\title{
Game Theoretic Models of Sustainable Management in Marketing Networks *
}

\author{
Movlatkhan T. Agieva ${ }^{1}$, Alexei V. Korolev ${ }^{2}$ and Guennady A. \\ Ougolnitsky ${ }^{3}$ \\ 1 Ingush State University \\ Magistralnaya St. 39, Nazran, 386132, Russia \\ E-mail: agieva25@mail.ru \\ 2 National Research University Higher School of Economics at St. Petersburg \\ Khantemirovskaya St. 3A, St. Petersburg, Russia \\ E-mail: danitschi@gmail.com \\ 3 Southern Federal University, J.I. Vorovich Institute of Mathematics, \\ Mechanics and Computer Sciences \\ Milchakov St. 8a, Rostov-on-Don, 344090, Russia \\ E-mail: gaugolnickiy@sfedu.ru
}

\begin{abstract}
Difference and differential Stackelberg games of opinion control on marketing networks are considered. The principal allocates financial resources to the firms for marketing purposes. It is supposed that the structure of a target audience described by a weighted directed graph is already determined in the stage of network analysis, and marketing control actions are applied only to the members of strong subgroups (opinion leaders). Conditions of homeostasis (phase constraints) which reflect the requirements of sustainable management are introduced additionally. The Stackelberg equilibria are found analytically. It is shown that the interests of the principal and the firms are completely compatible.
\end{abstract}

Keywords: difference Stackelberg games, differential Stackelberg games, marketing networks, sustainable management.

\section{Introduction}

The basic model of influence in a social network is a weighted directed graph where vertices represent the members of the network (basic agents), and arcs describe their mutual influence. Each vertex is ascribed a real value (an opinion of this agent) which is a function of time, and each arc is ascribed a constant real value (weight) which characterizes a degree of influence of an agent to another (or trust of the latter to the former). Besides, there are one or several influence agents (firms) which exert impact to the basic agents in their (influence agents) interests (Chkhartishvili et al., 2019). An approach close to ours is used in (Sedakov and Zhen, 2019; Zhen, 2019).

This paper represents formulations and solutions of the game theoretic models of opinion control in social groups with a given network structure of the interactions. The models are interpreted in (not unique) marketing terms. It is supposed that there is a coordinating body which determines the firms' marketing budgets. Thus, the Stackelberg games of the type "one principal - several agents" are considered. It is also supposed that in the stage of network analysis the target audience is already segmented into strong subgroups (opinion leaders) and satellites

\footnotetext{
${ }^{\star}$ The work was supported by the Russian Science Foundation, project N 17-19-01038.
} 
(Agieva et al., 2019). Then the control actions are restricted by the set of members of the strong subgroups that reduces the marketing expenditures essentially.

The objective of the control impact is maximization of the sum of opinions of all agents at the whole period from $\mathrm{t}=1$ till $\mathrm{t}=\mathrm{T}$. The impact in closed-loop strategies is exerted to the current opinions of the members of strong subgroups. Accordingly to the requirements of sustainable management, the state vector of the controlled dynamical system must belong to a certain domain of the space of states (homeostasis conditions) (Ougolnitsky, 2011).

The dynamics of the controlled system (opinions of the agents) is described by difference of differential equations. Respectively, the payoff functionals of the principal and the agents are or sum, or integral of their current payoff functions. An introduction of the discrete or continuous time are complementary approaches which permit to reflect different effects in real systems, and it is rational to use both types of the models. Thus, both difference and differential Stackelberg games with several agents are studied. The best response of the agents is defined as a Nash equilibrium in their game in strategic form. The difference models are analyzed by induction on the number of game periods, and the differential models are studied by means of the Hamilton-Jacobi-Bellman equations. For the consideration of budget constraints the Lagrange multipliers method is used.

The key problem in hierarchical systems is the coordination of interests of different control levels. The most widespread formulation of this problem is a comparison of the socially optimal outcome of the game with outcomes of the egoistic behavior of the players (the problem of inefficiency of equilibria) (Nisan et al., 2007). In this paper the index of system compatibility is used for the characteristic of the coordination of interests. It is a fraction in which the numerator is a value of the principal's payoff in the worst Nash equilibrium in the game of the agents, and the denominator is the value of her globally maximal payoff (Sukhinov et al., 2020).

For the solution of the problems with the homeostasis condition we use the following approach. First, we solve a basic optimization problem without this condition and find the optimal strategies of the players. In the basic model both principal and agents maximize the summary opinions of the target audience, and the principal determines the marketing budgets of the agents. Given the optimal strategies and the homeostasis condition (the sum of the state variables should not exceed a given value $x^{*}$ ), we suppose that the influence matrix $A$ is stochastic (from the right). This assumption permits to abstract from the interaction of the basic agents. In this case the multiplication by the matrix $A$ from the left does not change the sum of components of the state vector. Then the algorithm consists in optimal increasing of the sum of the state variables up to the level $x^{*}$, and then to do nothing unless the period of consideration finishes.

However, this assumption is too strong. The standard assumption in the control problems on networks is that the matrix $A$ is stochastic from the left. The matrix stochastic from the left has also a Frobenius eigenvalue equal to one, and a positive Frobenius eigenvector corresponding to this value. By means of the diagonal matrix $\Xi$ with the components of this Frobenius eigenvector on the main diagonal, we can perform the conjunction operation and move from the initial influence matrix $A$ to the similar matrix $P$ which is stochastic from the right. Making a substitution of the state variables by the transform matrix $\Xi$, we reduce the initial problem with the influence matrix $A$ to the problem with the stochastic matrix $P$ in the new 
coordinates. Solving this problem and making the inverse transform of the variables, we receive the solution of the initial problem.

The payment for this transform consists in a small change of the problem formulation. The initial homeostasis condition $\sum_{j=1}^{n} x_{j} \leq x^{*}$ takes the form $\sum_{j=1}^{n} z_{j} x_{j} \leq$ $x^{*}$, where $z_{j}$ are components of the Frobenius eigenvector of the matrix $A$. Though these two formulations are not equivalent, they do not differ essentially. In fact, in the initial formulation we could take instead of $x^{*}$ the value $x / \sum_{j=1}^{n} z_{j}$, and the degree of the resulting values of the state variables remains the same. Besides, now we have to maximize not simply the sum $\sum_{j=1}^{n} x_{j}$ of the values of state variables but the projection of the demand vector to the Frobenius eigenvector of the matrix $A$. In other words, we maximize only the summary demand $\sum_{j=1}^{n} z_{j} x_{j}$ along a general direction of the mutual influences of the agents as it is done with a standard good in the Leontief-Sraffa model (Leontief, 1987; Sraffa, 1960; Sraffa, 1962).

We use this approach both in the discrete and continuous time. Respectively, the paper consists of four parts. In the Sections 2 and 3 we study difference Stackelberg game theoretic models of opinion control in which the principal should provide that a given limit value of the summary opinion not be exceeded. In the Section 2 the problem is solved for the stochastic influence matrix A, and in the Section 3 this strong assumption is substituted by a weaker standard assumption that the matrix A is stochastic from the left. The values of the system compatibility index are calculated. They are equal to one that witnesses about an ideal coordination of the modeled system. It appears that for the principal it is not advantageous to allocate to the agents more resources than they need from the point of view of their rational behavior. In the Sections 4 and 5 we analyze differential Stackelberg game theoretic models of opinion control with the homeostasis condition by the completely similar scheme. The Section 6 concludes.

\section{Difference Stackelberg Game with a Constraint on the Sum of State Variables. Case of the Stochastic (from the Right) Matrix $A$}

\subsection{The Problem Statement}

The model has the form

$$
\begin{gathered}
J_{0}=\sum_{t=0}^{T} \delta^{t}\left[\sum_{j=1}^{n} x_{j}^{t}-\sum_{i=1}^{m} r_{i}^{t}\right] \rightarrow \max , \\
r_{i}^{t} \geq 0, \quad \sum_{i=1}^{m} r_{i}^{t} \leq R, \quad t=0,1,2, \ldots, T, \quad i=1,2, \ldots, m, \\
x_{*} \leq \sum_{j=1}^{n} x_{j}^{t} \leq R, \quad t=0,1,2, \ldots, T-1, T, \\
J_{i}=\sum_{t=0}^{T} \delta^{t}\left[\sum_{j=1}^{n}\left(x_{j}^{t}-s_{j}^{i} u_{j}^{i, t}\right)\right] \rightarrow \max , \\
\sum_{j=1}^{n} u_{j}^{i, t} \leq r_{i}^{t}, \quad u_{j}^{i, t} \geq 0, \quad t=0,1,2, \ldots, T, \quad i=1,2, \ldots, m,
\end{gathered}
$$




$$
\begin{gathered}
x_{j}^{t+1}=\sum_{i=1}^{m} b_{j}^{i} \sqrt{u_{j}^{i, t}}+\sum_{l=1}^{n} a_{l j} x_{l}^{t}, \quad x_{j}^{0}=x_{j 0}, \quad j=1,2, \ldots, n, \\
s_{j}^{i}=\left\{\begin{array}{lll}
1, & \text { if } & b_{j}^{i}>0, \\
0, & \text { if } & b_{j}^{i}=0 .
\end{array}\right.
\end{gathered}
$$

Here $n$ - a number of basic agents (a number of target audience), $m$ - a number of control agents (competing firms), $R$ - a total marketing budget of the leader, $T$ a length of the game, $J_{0}, J_{i}$ - the payoff functionals of the leader and the followers (control agents) respectively, $r_{i}^{t}$ - a marketing budget allocated to the $i$-th follower by the leader in the moment (discrete period) of time $t, x_{j}^{t}$ - an opinion of the $j$-th basic agent in the moment $t, r_{i}^{t}$ - expenditures of the $i$-th control agent for the marketing impact (advertizing and so on) to the $j$-th basic agent in the moment $t$, $a_{i j}$ - a coefficient of influence of the $i$-th basic agent to the $j$-th basic agent, $b_{j}^{i}-$ a coefficient of influence of the $i$-th control agent to the $j$-th basic agent, $\delta$ denotes a discount factor, i.e. $\delta=e^{-\rho}$. As different firms can exert influence to different members of the strong subgroups, we simply assume that if the $i$-th firm (control agent) does not influence to the $j$-th basic agent then $b_{j}^{i}=0$. Denote by $A$ a matrix of the coefficients of influence among basic agents, i.e. $A=\left\{a_{i j}\right\}_{i=1,2, \ldots, n, j=1,2, \ldots, n}$, $A^{\tau}$ - a transposed matrix of influences, $X^{t}$ - a column vector of the values of state variables (opinions) in the moment $t, \varepsilon-$ a row vector of the dimension $n$ formed by units, $I$ - the unit $n \times n$-matrix.

Assume that matrix of influences of the basic agents $A$ is stochastic (from the right), i.e.

$$
\sum_{j=1}^{n} a_{i j}=1, \quad i=1,2, \ldots, n .
$$

In this case an action of the matrix $A^{\tau}$ from the left on any vector does not change the sum of its components. Thus,

$$
\begin{gathered}
\varepsilon\left(A^{\tau}\right)^{T} X^{0}=\varepsilon\left(A^{\tau}\right)^{T-1} X^{0}=\cdots=\varepsilon A^{\tau} X^{0}=\varepsilon X^{0}, \\
A_{j}=A_{j}^{2}=\cdots=A_{j}^{T}=1, \quad j=1,2, \ldots, n,
\end{gathered}
$$

where $\varepsilon$ is a row vector of $n$ units.

\subsection{Solving the $i$-th Firm's Problem}

Consider the problem of the $i$-th firm (4) - (7). In the one-period (without consideration of the zero period) game each $i$-th firm solves the problem

$$
\begin{gathered}
\sum_{j=1}^{n} x_{j}^{0}-\sum_{j=1}^{n} s_{j}^{i} u_{j}^{i, 0}+\delta \sum_{j=1}^{n} x_{j}^{i, 1}= \\
=\sum_{j=1}^{n} x_{j}^{0}-\sum_{j=1}^{n} s_{j}^{i} u_{j}^{i, 0}+\delta \sum_{j=1}^{n} \sum_{i=1}^{m} b_{j}^{i} \sqrt{u_{j}^{i, 0}}+\delta \sum_{j=1}^{n} \sum_{l=1}^{n} a_{l j} x_{l}^{0} \underset{u_{j}^{i, 0},{ }_{j=1,2, \ldots, n}}{\longrightarrow} \max
\end{gathered}
$$

with constraint

$$
\sum_{j=1}^{n} u_{j}^{i, 0} \leq r_{i}^{0}
$$


Optimizing by the Lagrange multipliers method, we receive the relations:

$$
\frac{b_{j_{1}}^{i}}{b_{j_{2}}^{i}}=\sqrt{\frac{u_{j_{1}}^{i, 0}}{u_{j_{2}}^{i, 0}}}
$$

for any agents $j_{1}$ and $j_{2}$ impacted by the $i$-th firm, therefore

$$
u_{j_{2}}^{i, 0}=u_{j_{1}}^{i, 0}\left(\frac{b_{j_{2}}^{i}}{b_{j_{1}}^{i}}\right)^{2} .
$$

Denote $\sum_{j=1}^{n} u_{j}^{i, 0}$ by $R_{i}^{0}$, then we have:

$$
\sum_{j=1}^{n} u_{j}^{i, 0}=\frac{u_{j_{1}}^{i, 0}}{\left(b_{j_{1}}^{i}\right)^{2}} \sum_{j=1}^{n}\left(b_{j}^{i}\right)^{2}=R_{i}^{0}
$$

and, therefore,

$$
u_{j}^{i, 0}=\frac{\left(b_{j}^{i}\right)^{2} R_{i}^{0}}{\sum_{j=1}^{n}\left(b_{j}^{i}\right)^{2}} .
$$

The substitution of (9) into (8) gives

$$
\sum_{j=1}^{n} x_{j}^{0}-R_{i}^{0}+\delta \sum_{i=1}^{m} \sqrt{R_{i}^{0} \sum_{j=1}^{n}\left(b_{j}^{i}\right)^{2}}+\delta \sum_{j=1}^{n} \sum_{l=1}^{n} a_{l j} x_{l}^{0} \underset{u_{j}^{i, 0},{ }_{j=1,2, \ldots, n}}{\longrightarrow} \max .
$$

A non-conditional optimization by $R_{i}^{0}$ implies

$$
\left(R_{i}^{0}\right)_{\max }=\frac{\delta^{2}}{4} \sum_{j=1}^{n}\left(b_{j}^{i}\right)^{2} .
$$

Thus, the optimal strategy of the $i$-th firm is

$$
u_{j}^{i, 0}=\frac{\left(b_{j}^{i}\right)^{2}}{\sum_{j=1}^{n}\left(b_{j}^{i}\right)^{2}} \min \left\{\frac{\delta^{2}}{4} \sum_{j=1}^{n}\left(b_{j}^{i}\right)^{2}, r_{i}^{0}\right\} .
$$

Consider a two-period game. Each firm $i$ solves the problem

$$
\sum_{j=1}^{n}\left(x_{j}^{0}-s_{j}^{i} u_{j}^{i, 0}\right)+\delta \sum_{j=1}^{n}\left(x_{j}^{1}-s_{j}^{i} u_{j}^{i, 1}\right)+\delta^{2} \sum_{j=1}^{n} x_{j}^{2} \underset{u_{j}^{i, 0}, \underset{j=1,2, \ldots, n}{\longrightarrow} \max }{\longrightarrow}
$$

with constraint

$$
\sum_{j=1}^{n} u_{j}^{i, 0} \leq r_{i}^{0}
$$

where $u_{j}^{i, 1}, j=1,2, \ldots, n$, is the solution of the one-period game. We have

$$
x_{j}^{1}=\sum_{i=1}^{m} b_{j}^{i} \sqrt{u_{j}^{i, 0}}+\sum_{l=1}^{n} a_{l j} x_{l}^{0},
$$




$$
\begin{gathered}
x_{j}^{2}=\sum_{i=1}^{m} b_{j}^{i} \sqrt{u_{j}^{i, 1}}+\sum_{l=1}^{n} a_{l j} x_{l}^{1}=\sum_{i=1}^{m} b_{j}^{i} \sqrt{u_{j}^{i, 1}}+\sum_{l=1}^{n} a_{l j}\left(\sum_{i=1}^{m} b_{j}^{i} \sqrt{u_{j}^{i, 0}}+\sum_{p=1}^{n} a_{p l} x_{p}^{0}\right)= \\
=\sum_{i=1}^{m} b_{j}^{i} \sqrt{u_{j}^{i, 1}}+\sum_{l=1}^{n} a_{l j} \sum_{i=1}^{m} b_{l}^{i} \sqrt{u_{l}^{i, 0}}+\left[\left(A^{\tau}\right)^{2} X^{0}\right]_{j} .
\end{gathered}
$$

Substituting (12) and (13) into (11) we receive

$$
\begin{gathered}
\sum_{j=1}^{n}\left(x_{j}^{0}-s_{j}^{i} u_{j}^{i, 0}\right)+\delta \sum_{j=1}^{n}\left(\sum_{i=1}^{m} b_{j}^{i} \sqrt{u_{j}^{i, 0}}+\sum_{l=1}^{n} a_{l j} x_{l}^{0}-s_{j}^{i} u_{j}^{i, 1}\right)+ \\
+\delta^{2} \sum_{j=1}^{n} \sum_{i=1}^{m} b_{j}^{i} \sqrt{u_{j}^{i, 1}}+\delta^{2} \sum_{j=1}^{n} \sum_{l=1}^{n} a_{l j} \sum_{i=1}^{m} b_{l}^{i} \sqrt{u_{l}^{i, 0}}+\delta^{2} \varepsilon\left(A^{\tau}\right)^{2} X^{0} \underset{u_{j}^{i, 0}, \underset{j=1,2, \ldots, n}{\longrightarrow} \max ,}{\longrightarrow}
\end{gathered}
$$

or, denote by $A_{j}$ the sum of the elements of the $j$-th row of the influence matrix $A$,

$$
\begin{gathered}
\sum_{j=1}^{n}\left(x_{j}^{0}-s_{j}^{i} u_{j}^{i, 0}\right)+\delta \sum_{j=1}^{n}\left(\sum_{i=1}^{m} b_{j}^{i} \sqrt{u_{j}^{i, 0}}+\sum_{l=1}^{n} a_{l j} x_{l}^{0}-s_{j}^{i} u_{j}^{i, 1}\right)+ \\
+\delta^{2} \sum_{j=1}^{n} \sum_{i=1}^{m} b_{j}^{i} \sqrt{u_{j}^{i, 1}}+\delta^{2} \sum_{j=1}^{n} \sum_{i=1}^{m} A_{j} b_{j}^{i} \sqrt{u_{j}^{i, 0}}+\delta^{2} \varepsilon\left(A^{\tau}\right)^{2} X^{0} \underset{u_{j}^{i, 0},{ }_{j=1,2, \ldots, n}}{\longrightarrow} \max ,
\end{gathered}
$$

Maximizing (14) with constraint

$$
\sum_{j=1}^{n} u_{j}^{i, 0} \leq r_{i}^{0}
$$

by the Lagrange multipliers method, we receive the relations

$$
\frac{\left(1+\delta A_{j_{2}}\right) b_{j_{2}}^{i}}{\left(1+\delta A_{j_{1}}\right) b_{j_{1}}^{i}}=\sqrt{\frac{u_{j_{2}}^{i, 0}}{u_{j_{1}}^{i, 0}}}
$$

for any agents $j_{1}$ and $j_{2}$ impacted by the $i$-th firm, therefore

$$
u_{j_{2}}^{i, 0}=u_{j_{1}}^{i, 0}\left(\frac{\left(1+\delta A_{j_{2}}\right) b_{j_{2}}^{i}}{\left(1+\delta A_{j_{1}}\right) b_{j_{1}}^{i}}\right)^{2}
$$

Denote $\sum_{j=1}^{n} u_{j}^{i, 0}$ by $R_{i}^{0}$, then receive:

$$
\sum_{j=1}^{n} u_{j}^{i, 0}=\frac{u_{j_{1}}^{i, 0}}{\left[\left(1+\delta A_{j_{1}}\right) b_{j_{1}}^{i}\right]^{2}} \sum_{j=1}^{n}\left[\left(1+\delta A_{j}\right) b_{j}^{i}\right]^{2}=R_{i}^{0}
$$

and, therefore,

$$
u_{j}^{i, 0}=\frac{\left[\left(1+\delta A_{j}\right) b_{j}^{i}\right]^{2} R_{i}^{0}}{\sum_{j=1}^{n}\left[\left(1+\delta A_{j}\right) b_{j}^{i}\right]^{2}} .
$$


Substituting (15) into (14) and choosing only the terms with $u_{j}^{i, 0}$, we receive

$$
\begin{gathered}
-R_{i}^{0}+\delta \frac{\sum_{j=1}^{n} \sum_{i=1}^{m} b_{j}^{i} \sqrt{u_{j}^{i, 0}} \sqrt{R_{i}^{0}}}{\sqrt{\sum_{j=1}^{n}\left[b_{j}^{i}\left(1+\delta A_{j}\right)\right]^{2}}}+\delta^{2} \frac{\sum_{j=1}^{n} \sum_{i=1}^{m} b_{j}^{i} A_{j} \sqrt{u_{j}^{i, 0}} \sqrt{R_{i}^{0}}}{\sqrt{\sum_{j=1}^{n}\left[b_{j}^{i}\left(1+\delta A_{j}\right)\right]^{2}}}= \\
=-R_{i}^{0}+\delta \sum_{i=1}^{m} \sqrt{R_{i}^{0} \sum_{j=1}^{n}\left[b_{j}^{i}\left(1+\delta A_{j}\right)\right]^{2}} .
\end{gathered}
$$

A non-conditional maximization of (16) by $R_{i}^{0}$ gives:

$$
\left(R_{i}^{0}\right)_{\max }=\frac{\delta^{2}}{4} \sum_{j=1}^{n}\left[b_{j}^{i}\left(1+\delta A_{j}\right)\right]^{2} .
$$

Thus, the optimal strategy of the $i$-th firm is

$$
u_{j}^{i, 0}=\frac{\left[b_{j}^{i}\left(1+\delta A_{j}\right)\right]^{2}}{\sum_{j=1}^{n}\left[b_{j}^{i}\left(1+\delta A_{j}\right)\right]^{2}} \min \left\{\frac{\delta^{2}}{4} \sum_{j=1}^{n}\left[b_{j}^{i}\left(1+\delta A_{j}\right)\right]^{2}, r_{i}^{0}\right\} .
$$

Now consider the $T$-period game. Denote

$$
\left(\sum_{i=1}^{m} b_{j}^{i} \sqrt{u_{j}^{i, t}}\right)_{j=1}^{n}=\beta^{t}, \quad t=0,1,2, \ldots T .
$$

Then we have

$$
\begin{gathered}
X^{1}=A^{\tau} X^{0}+\beta^{0}, \\
X^{2}=\left(A^{\tau}\right)^{2}+A^{\tau} \beta^{0}+\beta^{1},
\end{gathered}
$$

and iterations by $t$ give

$$
X^{t}=\left(A^{\tau}\right)^{t} X^{0}+\left(A^{\tau}\right)^{t-1} \beta^{0}+\left(A^{\tau}\right)^{t-2} \beta^{1}+\cdots+A^{\tau} \beta^{t-2}+\beta^{t-1} .
$$

Each $i$-th firm solves the problem

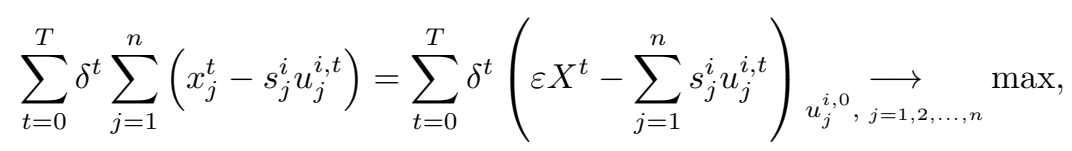

with constraint

$$
\sum_{j=1}^{n} u_{j}^{i, 0} \leq r_{i}^{0}
$$

where $u_{j}^{i, 1}, u_{j}^{i, 2}, \ldots, u_{j}^{i, T-1}(j=1,2, \ldots, n)$ are the respective solutions of the $(T-1)$ period, $(T-2)$-period, ..., one-period problems.

Choosing in (19) only the terms whith variables $u_{j}^{i, 0}, j=1,2, \ldots, n$, we receive the expression

$$
-\sum_{j=1}^{n} s_{j}^{i} u_{j}^{i, 0}+\varepsilon\left[\delta I+\delta^{2} A^{\tau}+\cdots+\delta^{T}\left(A^{\tau}\right)^{T-1}\right] \beta^{0} .
$$


Maximizing (20) with the constraint

$$
\sum_{j=1}^{n} u_{j}^{i, 0} \leq r_{i}^{0}
$$

by the Lagrange multipliers method, we receive the relations

$$
\frac{\left(1+\delta A_{j_{2}}+\cdots+\delta^{T-1} A_{j_{2}}^{T-1}\right) b_{j_{2}}^{i}}{\left(1+\delta A_{j_{1}}+\cdots+\delta^{T-1} A_{j_{1}}^{T-1}\right) b_{j_{1}}^{i}}=\sqrt{\frac{u_{j_{2}}^{i, 0}}{u_{j_{1}}^{i, 0}}}
$$

for any agents $j_{1}$ and $j_{2}$ influenced by the $i$-th firm, where $A_{j}^{t}$ denotes the sum of elements of the $j$-th row of the $t$-th power of the influence matrix, $t=1,2, \ldots, T-1$. Denote as earlier the sum $\sum_{j=1}^{n} u_{j}^{i, 0}$ by $R_{i}^{0}$, we receive

$$
u_{j}^{i, 0}=\frac{\left(b_{j}^{i} \sum_{t=0}^{T-1} \delta^{t} A_{j}^{t}\right)^{2} R_{i}^{0}}{\sum_{j=1}^{n}\left(b_{j}^{i} \sum_{t=0}^{T-1} \delta^{t} A_{j}^{t}\right)^{2}} .
$$

A substitution of (22) into (20) with consideration of (17) gives

$$
\begin{gathered}
-\sum_{j=1}^{n} s_{j}^{i} u_{j}^{i, 0}+\sum_{i=1}^{m} \sum_{j=1}^{n} \delta b_{j}^{i} \sum_{t=0}^{T-1} \delta^{t} A_{j}^{t} \frac{b_{j}^{i} \sum_{t=0}^{T-1} \delta^{t} A_{j}^{t} \sqrt{R_{i}^{0}}}{\sqrt{\sum_{j=1}^{n}\left(b_{j}^{i} \sum_{t=0}^{T-1} \delta^{t} A_{j}^{t}\right)^{2}}}= \\
=-R_{i}^{0}+\delta \sum_{i=1}^{m} \sqrt{R_{i}^{0} \sum_{j=1}^{n}\left(b_{j}^{i} \sum_{t=0}^{T-1} \delta^{t} A_{j}^{t}\right)^{2}} .
\end{gathered}
$$

Maximizing (23) by $R_{i}^{0}$ without restrictions, we receive

$$
1=\frac{\delta \sqrt{\sum_{j=1}^{n}\left(b_{j}^{i} \sum_{t=0}^{T-1} \delta^{t} A_{j}^{t}\right)^{2}}}{2 \sqrt{R_{i}^{0}}} .
$$

Therefore,

$$
\left(R_{i}^{0}\right)_{\max }=\frac{\delta^{2}}{4} \sum_{j=1}^{n}\left(b_{j}^{i} \sum_{t=0}^{T-1} \delta^{t} A_{j}^{t}\right)^{2} .
$$

Thus, with consideration of the constraint (21),

$$
u_{j}^{i, 0}=\frac{\left(b_{j}^{i} \sum_{t=0}^{T-1} \delta^{t} A_{j}^{t}\right)^{2}}{\sum_{j=1}^{n}\left(b_{j}^{i} \sum_{t=0}^{T-1} \delta^{t} A_{j}^{t}\right)^{2}} \min \left\{\frac{\delta^{2}}{4} \sum_{j=1}^{n}\left(b_{j}^{i} \sum_{t=0}^{T-1} \delta^{t} A_{j}^{t}\right)^{2}, r_{i}^{0}\right\} .
$$

Respectively, in the period $t(t=1,2, \ldots, T-1)$ we have

$$
u_{j}^{i, t}=\frac{\left(b_{j}^{i} \sum_{s=0}^{T-1-t} \delta^{s} A_{j}^{s}\right)^{2}}{\sum_{j=1}^{n}\left(b_{j}^{i} \sum_{s=0}^{T-1-t} \delta^{s} A_{j}^{s}\right)^{2}} \min \left\{\frac{\delta^{2}}{4} \sum_{j=1}^{n}\left(b_{j}^{i} \sum_{s=0}^{T-1-t} \delta^{s} A_{j}^{s}\right)^{2}, r_{i}^{t}\right\},
$$


where $r_{i}^{t}$ are the resources allocated to the $i$-th firm by the principal in the $t$ th period. As the principal in the period $t$ never chooses a value $r_{i}^{t}$ greater than $\frac{\delta^{2}}{4} \sum_{j=1}^{n}\left(b_{j}^{i} \sum_{s=0}^{T-1-t} \delta^{s} A_{j}^{s}\right)^{2}$, the expression (24) may be substituted by

$$
u_{j}^{i, t}=\frac{\left(b_{j}^{i} \sum_{s=0}^{T-1-t} \delta^{s} A_{j}^{s}\right)^{2} r_{i}^{t}}{\sum_{j=1}^{n}\left(b_{j}^{i} \sum_{s=0}^{T-1-t} \delta^{s} A_{j}^{s}\right)^{2}} .
$$

Considering the stochasticity of the matrix $A$, the expression (25) takes the form

$$
u_{j}^{i, t}=\frac{\left(b_{j}^{i} \sum_{s=0}^{T-1-t} \delta^{s} A_{j}^{s}\right)^{2} r_{i}^{t}}{\sum_{j=1}^{n}\left(b_{j}^{i} \sum_{s=0}^{T-1-t} \delta^{s} A_{j}^{s}\right)^{2}}=\frac{\left(b_{j}^{i} \sum_{s=0}^{T-1-t} \delta^{s}\right)^{2} r_{i}^{t}}{\sum_{j=1}^{n}\left(b_{j}^{i} \sum_{s=0}^{T-1-t} \delta^{s}\right)^{2}}=\frac{\left(b_{j}^{i}\right)^{2} r_{i}^{t}}{\sum_{j=1}^{n}\left(b_{j}^{i}\right)^{2}}
$$

and does not depend on the length of period $T$. Thus we have proved the statement.

Proposition 1. The optimal strategy of the $i$-th firm in problem (4)-(7) is

$$
u_{j}^{i, t}=\frac{\left(b_{j}^{i}\right)^{2} r_{i}^{t}}{\sum_{j=1}^{n}\left(b_{j}^{i}\right)^{2}} .
$$

\subsection{Solving the Principal's Problem}

The principal cannot decrease the value $\sum_{j=1}^{n} x_{j}$, and only increases it by allocation the resources between the firms. It follows from the problem formulation that $x_{*} \leq \sum_{j=1}^{n} x_{j}^{0}<x^{*}$, and the value $\sum_{j=1}^{n} x_{j}$ cannot become less than $x_{*}$. Then the principal's optimal strategy is evident. The principal solves the problem (1)-(3), (5)-(6).

It is clear that for the maximization of her objective function the principal must optimally increase the value $\sum_{j=1}^{n} x_{j}^{t}$ only up to the value $x^{*}$, after what she must cease the allocation of resources to the firms. Then the value $\sum_{j=1}^{n} x_{j}^{t}$ remains equal to $x^{*}$ until the end of the period of consideration. For the implementation of this strategy the principal already in the instant of time $t=0$ must determine the instant $t=H$, when the value $\sum_{j=1}^{n} x_{j}^{H}$ becomes greater than $x^{*}$ due to the respective strategy. At the instant $t=H-1$ the value of allocated resources should be reduced so that starting from the instant $t=H$ the value $\sum_{j=1}^{n} x_{j}^{t}$ be strictly equal to $x^{*}$.

Consider the $H$-period game. The principal solves the problem

$$
\sum_{t=0}^{H} \delta^{t}\left(\sum_{j=1}^{n} x_{j}^{t}-\sum_{i=1}^{m} r_{i}^{t}\right)=\sum_{t=0}^{H} \delta^{t}\left(\varepsilon X^{t}-\sum_{i=1}^{m} r_{i}^{t}\right)_{r_{i}^{0}, \underset{i=1,2, \ldots, m}{\longrightarrow} \max ,}^{\longrightarrow}
$$

with constraint

$$
\sum_{i=1}^{m} r_{i}^{0} \leq R
$$

where $r_{i}^{1}, r_{i}^{2}, \ldots, r_{i}^{H-1}(i=1,2, \ldots, m)$ are the respective solutions of the $(H-1)$ period, $(H-2)$-period, .... one-period problems, and

$$
u_{j}^{i, t}=\frac{\left(b_{j}^{i}\right)^{2} r_{i}^{t}}{\sum_{j=1}^{n}\left(b_{j}^{i}\right)^{2}}, \quad t=1,2, \ldots, H-1
$$


according to (26). Using the denotation (17) and decomposition (18), choosing in (27) only the terms that include $r_{i}^{0}$, and considering that $A_{j}^{t}=1$, we receive the expression

$$
-\sum_{i=1}^{m} r_{i}^{0}+\left(\delta+\delta^{2}+\cdots+\delta^{H}\right) \varepsilon \beta^{0}
$$

where

$$
\beta^{0}=\left(\sum_{i=1}^{m} b_{j}^{i} \sqrt{u_{j}^{i, 0}}\right)_{j=1}^{n}=\left(\sum_{i=1}^{m} \frac{\sqrt{r_{i}^{0}}\left(b_{j}^{i}\right)^{2}}{\sqrt{\sum_{j=1}^{n}\left(b_{j}^{i}\right)^{2}}}\right)_{j=1}^{n} .
$$

Substituting (29) into (28), we receive

$$
\begin{gathered}
-\sum_{i=1}^{m} r_{i}^{0}+\left(\delta+\delta^{2}+\cdots+\delta^{H}\right) \sum_{j=1}^{n} \sum_{i=1}^{m} \frac{\sqrt{r_{i}^{0}}\left(b_{j}^{i}\right)^{2}}{\sqrt{\sum_{j=1}^{n}\left(b_{j}^{i}\right)^{2}}}= \\
=-\sum_{i=1}^{m} r_{i}^{0}+\sum_{i=1}^{m} \sqrt{r_{i}^{0}} \sqrt{\sum_{j=1}^{n}\left(b_{j}^{i}\right)^{2}} \sum_{t=1}^{H} \delta^{t} .
\end{gathered}
$$

Maximizing (30) with constraint

$$
\sum_{i=1}^{m} r_{i}^{0} \leq R
$$

by the Lagrange multipliers method, we receive the relations

$$
\frac{\sum_{j=1}^{n}\left(b_{j}^{i_{2}}\right)^{2}}{\sum_{j=1}^{n}\left(b_{j}^{i_{1}}\right)^{2}}=\frac{r_{i_{2}}^{0}}{r_{i_{1}}^{0}}
$$

for any influence agents $i_{1}$ and $i_{2}$. Denote as earlier the sum $\sum_{i=1}^{m} r_{i}^{0}$ by $R^{0}$, we receive

$$
\sum_{i=1}^{m} r_{i}^{0}=\frac{\sum_{i=1}^{m} \sum_{j=1}^{n}\left(b_{j}^{i}\right)^{2} r_{i_{1}}^{0}}{\sum_{j=1}^{n}\left(b_{j}^{i_{1}}\right)^{2}}=R^{0}
$$

therefore

$$
r_{i}^{0}=\frac{\sum_{j=1}^{n}\left(b_{j}^{i}\right)^{2} R^{0}}{\sum_{i=1}^{m} \sum_{j=1}^{n}\left(b_{j}^{i}\right)^{2}} .
$$

Substituting the expression (31) into (30) we receive

$$
-R^{0}+\sqrt{R^{0}} \sum_{t=1}^{H} \delta^{t} \sqrt{\sum_{i=1}^{m} \sum_{j=1}^{n}\left(b_{j}^{i}\right)^{2}} .
$$

Maximizing the expression (32) by $R^{0}$ without constraints, we receive

$$
1=\frac{\delta \sqrt{\sum_{i=1}^{m} \sum_{j=1}^{n}\left(b_{j}^{i}\right)^{2}}}{2 \sqrt{R^{0}}} \sum_{t=0}^{H-1} \delta^{t}
$$


and

$$
\left(R^{0}\right)_{\max }=\frac{\delta^{2}}{4} \sum_{i=1}^{m} \sum_{j=1}^{n}\left(b_{j}^{i} \sum_{t=0}^{H-1} \delta^{t}\right)^{2} .
$$

Therefore, with consideration of the constraint $R^{0} \leq R$ the principal's optimal strategy is

$$
r_{i}^{0}=\frac{\sum_{j=1}^{n}\left(b_{j}^{i}\right)^{2}}{\sum_{i=1}^{m} \sum_{j=1}^{n}\left(b_{j}^{i}\right)^{2}} \min \left\{\frac{\delta^{2}}{4} \sum_{i=1}^{m} \sum_{j=1}^{n}\left(b_{j}^{i}\right)^{2}\left(\sum_{t=0}^{H-1} \delta^{t}\right)^{2}, R\right\} .
$$

Completely similar to (33) we receive for $t=1,2, \ldots, H-1$ :

$$
r_{i}^{t}=\frac{\sum_{j=1}^{n}\left(b_{j}^{i}\right)^{2}}{\sum_{i=1}^{m} \sum_{j=1}^{n}\left(b_{j}^{i}\right)^{2}} \min \left\{\frac{\delta^{2}}{4} \sum_{i=1}^{m} \sum_{j=1}^{n}\left(b_{j}^{i}\right)^{2}\left(\sum_{s=0}^{H-1-t} \delta^{s}\right)^{2}, R\right\}, i=1,2, \ldots, m .
$$

Using the denotation (17), decomposition (18) and the formula (26), we can write the value $\sum_{j=1}^{n} x_{j}^{H}$ in the $H$-period game, $t=0,1,2, \ldots, H$, where $H$ is exactly the instant when the value $\sum_{j=1}^{n} x_{j}^{t}$ first becomes equal to $x^{*}$, in the form

$$
\begin{gathered}
\varepsilon X^{H}=\varepsilon X^{0}+\varepsilon \beta^{0}+\varepsilon \beta^{1}+\cdots+\varepsilon \beta^{H-2}+\varepsilon \beta^{H-1}= \\
=\sum_{j=1}^{n} x_{j}^{0}+\sum_{i=1}^{m} \sqrt{r_{i}^{0} \sum_{j=1}^{n}\left(b_{j}^{i}\right)^{2}}+\cdots+\sum_{i=1}^{m} \sqrt{r_{i}^{t} \sum_{j=1}^{n}\left(b_{j}^{i}\right)^{2}}+\cdots+\sum_{i=1}^{m} \sqrt{r_{i}^{H-1} \sum_{j=1}^{n}\left(b_{j}^{i}\right)^{2}} .
\end{gathered}
$$

Substituting (34) into (35), we receive

$$
\begin{gathered}
\varepsilon X^{H}=\sum_{j=1}^{n} x_{j}^{0}+\sqrt{\sum_{i=1}^{m} \sum_{j=1}^{n}\left(b_{j}^{i}\right)^{2}} \min \left\{\frac{\delta}{2} \sqrt{\sum_{i=1}^{m} \sum_{j=1}^{n}\left(b_{j}^{i}\right)^{2}}\left(\sum_{s=0}^{H-1} \delta^{s}\right), \sqrt{R}\right\}+ \\
+\cdots+\sqrt{\sum_{i=1}^{m} \sum_{j=1}^{n}\left(b_{j}^{i}\right)^{2} \min \left\{\frac{\delta}{2} \sqrt{\sum_{i=1}^{m} \sum_{j=1}^{n}\left(b_{j}^{i}\right)^{2}}\left(\sum_{s=0}^{H-1-t} \delta^{s}\right), \sqrt{R}\right\}+\cdots+} \\
+\sqrt{\sum_{i=1}^{m} \sum_{j=1}^{n}\left(b_{j}^{i}\right)^{2}} \min \left\{\frac{\delta}{2} \sqrt{\sum_{i=1}^{m} \sum_{j=1}^{n}\left(b_{j}^{i}\right)^{2}}(1+\delta), \sqrt{R}\right\}+ \\
+\sqrt{\sum_{i=1}^{m} \sum_{j=1}^{n}\left(b_{j}^{i}\right)^{2}} \min \left\{\frac{\delta}{2} \sqrt{\sum_{i=1}^{m} \sum_{j=1}^{n}\left(b_{j}^{i}\right)^{2}}, \sqrt{R}\right\} .
\end{gathered}
$$

So, $t=H$ is the time instant when the value

$$
\Delta=\sqrt{\sum_{i=1}^{m} \sum_{j=1}^{n}\left(b_{j}^{i}\right)^{2}} \min \left\{\frac{\delta}{2} \sqrt{\sum_{i=1}^{m} \sum_{j=1}^{n}\left(b_{j}^{i}\right)^{2}}, \sqrt{R}\right\}+
$$




$$
\begin{gathered}
+\sqrt{\sum_{i=1}^{m} \sum_{j=1}^{n}\left(b_{j}^{i}\right)^{2}} \min \left\{\frac{\delta}{2} \sqrt{\sum_{i=1}^{m} \sum_{j=1}^{n}\left(b_{j}^{i}\right)^{2}}(1+\delta), \sqrt{R}\right\}+\cdots+ \\
+\sqrt{\sum_{i=1}^{m} \sum_{j=1}^{n}\left(b_{j}^{i}\right)^{2} \min \left\{\frac{\delta}{2} \sqrt{\sum_{i=1}^{m} \sum_{j=1}^{n}\left(b_{j}^{i}\right)^{2}}\left(\sum_{s=0}^{H-1-t} \delta^{s}\right), \sqrt{R}\right\}+\cdots+} \\
+\sqrt{\sum_{i=1}^{m} \sum_{j=1}^{n}\left(b_{j}^{i}\right)^{2}} \min \left\{\frac{\delta}{2} \sqrt{\sum_{i=1}^{m} \sum_{j=1}^{n}\left(b_{j}^{i}\right)^{2}}\left(\sum_{s=0}^{H-1} \delta^{s}\right), \sqrt{R}\right\}
\end{gathered}
$$

first becomes greater or equal than $x^{*}-\sum_{j=1}^{n} x_{j}^{0}$ (if it is possible for a $t \leq T$ ). In this case denote

$$
\Delta_{1}=x^{*}-\sum_{j=1}^{n} x_{j}^{0}-\left(\Delta-\sqrt{\sum_{i=1}^{m} \sum_{j=1}^{n}\left(b_{j}^{i}\right)^{2}} \min \left\{\frac{\delta}{2} \sqrt{\sum_{i=1}^{m} \sum_{j=1}^{n}\left(b_{j}^{i}\right)^{2}}, \sqrt{R}\right\}\right) .
$$

It is clear that the summary amount of resources allocated by the principal between the firms in the instant $H-1$ so that in the instant $H$ the sum of the values of state variables be strictly equal to $x^{*}$, must be equal to

$$
\tilde{R}=\frac{\left(\Delta_{1}\right)^{2}}{\sum_{i=1}^{m} \sum_{j=1}^{n}\left(b_{j}^{i}\right)^{2}}
$$

We obtain the statement.

Proposition 2. The principal has in the problem (1)-(3), (5)-(6) the following optimal strategy. When $0 \leq t \leq H-2$

$$
r_{i}^{t}=\frac{\sum_{j=1}^{n}\left(b_{j}^{i}\right)^{2}}{\sum_{i=1}^{m} \sum_{j=1}^{n}\left(b_{j}^{i}\right)^{2}} \min \left\{\frac{\delta^{2}}{4} \sum_{i=1}^{m} \sum_{j=1}^{n}\left(b_{j}^{i}\right)^{2}\left(\sum_{s=0}^{H-1-t} \delta^{s}\right)^{2}, R\right\}
$$

when $t=H-1$

$$
r_{i}^{H-1}=\frac{\sum_{j=1}^{n}\left(b_{j}^{i}\right)^{2}}{\sum_{i=1}^{m} \sum_{j=1}^{n}\left(b_{j}^{i}\right)^{2}} \tilde{R}
$$

and when $H \leq t \leq T$

$$
r_{i}^{t}=0, \quad i=1,2, \ldots, m
$$

Corollary 1. The maximal guaranteed payoff of the principal is equal to

$$
\begin{gathered}
\left(\sum_{s=0}^{H-1} \delta^{s}\right) \sum_{j=1}^{n} x_{j}^{0}+\left(\sum_{s=1}^{H-1} \delta^{s}\right) \sqrt{R^{0} \sum_{i=1}^{m} \sum_{j=1}^{n}\left(b_{j}^{i}\right)^{2}}-R^{0}+ \\
+\left(\sum_{s=2}^{H-1} \delta^{s}\right) \sqrt{R^{1} \sum_{i=1}^{m} \sum_{j=1}^{n}\left(b_{j}^{i}\right)^{2}}-\delta R^{1}+\cdots+
\end{gathered}
$$




$$
\begin{gathered}
+\left(\sum_{s=t+1}^{H-1} \delta^{s}\right) \sqrt{R^{t} \sum_{i=1}^{m} \sum_{j=1}^{n}\left(b_{j}^{i}\right)^{2}}-\delta^{t} R^{t}+\cdots+ \\
+\delta^{H-1} \sqrt{R^{H-2} \sum_{i=1}^{m} \sum_{j=1}^{n}\left(b_{j}^{i}\right)^{2}}-\delta^{H-2} R^{H-2}-\delta^{H-1} \tilde{R}+x^{*} \sum_{h=H}^{T} \delta^{h},
\end{gathered}
$$

where

$$
\begin{gathered}
R^{0}=\min \left\{\frac{\delta^{2}}{4} \sum_{i=1}^{m} \sum_{j=1}^{n}\left(b_{j}^{i}\right)^{2}\left(\sum_{s=0}^{H-1} \delta^{s}\right)^{2}, R\right\}, \\
R^{1}=\min \left\{\frac{\delta^{2}}{4} \sum_{i=1}^{m} \sum_{j=1}^{n}\left(b_{j}^{i}\right)^{2}\left(\sum_{s=0}^{H-2} \delta^{s}\right)^{2}, R\right\}, \ldots, \\
R^{t}=\min \left\{\frac{\delta^{2}}{4} \sum_{i=1}^{m} \sum_{j=1}^{n}\left(b_{j}^{i}\right)^{2}\left(\sum_{s=0}^{H-1-t} \delta^{s}\right)^{2}, R\right\}, \ldots, \\
R^{H-2}=\min \left\{\frac{\delta^{2}}{4} \sum_{i=1}^{m} \sum_{j=1}^{n}\left(b_{j}^{i}\right)^{2}(1+\delta)^{2}, R\right\},
\end{gathered}
$$

by $R^{t}$ is denoted the sum $\sum_{i=1}^{m} r_{i}^{t}, t=0,1,2, \ldots, H-2$, and the value $\tilde{R}$ is determined by the expression (37).

Proof. It can be seen from (34).

\subsection{System Compatability Index}

Denote the set of collections of equilibrium strategies of firms provided that principal's strategy is $\left\{r_{i}\right\}_{i=1}^{m}$ by $N E\left(\left\{r_{i}\right\}_{i=1}^{m}\right)$. Calculate the system compatibility index for the considered problem:

$$
S C I=\frac{\max _{\left\{r_{i}\right\}_{i=1}^{m}} \min _{\left\{u_{j}^{i}\right\}_{i=1 ; j=1}^{m ;} \in N E\left(\left\{r_{i}\right\}_{i=1}^{m}\right)} J_{0}\left(\left\{r_{i}\right\}_{i=1}^{m},\left\{u_{j}^{i}\right\}_{i=1 ; j=1}^{m ;}\right)}{\max _{\left\{r_{i}\right\}_{i=1}^{m}} \max _{\left\{u_{j}^{i}\right\}_{i=1 ; j=1}^{m ;} J_{0}} J_{0}\left(\left\{r_{i}\right\}_{i=1}^{m},\left\{u_{j}^{i}\right\}_{i=1 ; j=1}^{m ;}\right)} .
$$

This index shows the measure of compatability of system. The numerator of (43) is the payoff of the principal in the case if the firms choose the most unfavorable for principal equilibrium strategies. The denumerator of (43) is the payoff of the principal in the case if the firms choose the most favorable for principal strategies.

Given the principal's strategy the set of equilibrium strategies of each agent is one-element in our case, so the numerator of the expression (43) is equal to (38). For calculation of the denominator in the expression (43) let us assume that each agent maximizes not his payoff but the principal's payoff given her strategy, i.e. the agent solves the problem

$$
\tilde{J}_{i}=\sum_{t=0}^{T} \delta^{t} \sum_{j=1}^{n} x_{j}^{t} \rightarrow \max ,
$$

with constraint (3) and (5) - (6). 
If $\sum_{j=1}^{n} x_{j}^{0} \geq x^{*}$ then the optimal strategy of each firm is evident: it does not invest to the advertisement. Moreover, it is impossible because in this case the principal does not allocate any financial resources to the firms, i.e. then $u_{j}^{i, t}=0$.

Let $\sum_{j=1}^{n} x_{j}^{0}<x^{*}$. Unlike the principal, any agent is unable to estimate a priori the number of time periods $H$ which is required for the sum of values of the state variables becomes equal to $x^{*}$, but it is redundant because the optimal strategies of the firms do not depend on the game's length.

Consider the $H$-period game. Denote as earlier

$$
\left(\sum_{i=1}^{m} b_{j}^{i} \sqrt{u_{j}^{i, t}}\right)_{j=1}^{n}=\beta^{t}, \quad t=0,1,2, \ldots H-1 .
$$

Then

$$
\begin{gathered}
X^{1}=X^{0}+\beta^{0}, \\
X^{2}=X^{0}+\beta^{0}+\beta^{1},
\end{gathered}
$$

and so on, the iterations by $t$ give

$$
X^{t}=X^{0}+\beta^{0}+\beta^{1}+\cdots+\beta^{t-2}+\beta^{t-1} .
$$

Each $i$-th firm solves the problem

$$
\sum_{t=0}^{T} \delta^{t} \sum_{j=1}^{n} x_{j}^{t}=\sum_{t=0}^{T} \delta^{t} \varepsilon X^{t} \underset{u_{j}^{i, 0},{ }_{j=1,2, \ldots, n}}{\longrightarrow} \max ,
$$

with constraint

$$
\sum_{j=1}^{n} u_{j}^{i, 0} \leq r_{i}^{0}
$$

where $u_{j}^{i, 1}, u_{j}^{i, 2}, \ldots, u_{j}^{i, H-1}(j=1,2, \ldots, n)$ are the respective solutions of the $(H-$ 1)-period, $(H-2)$-period, ..., one-period problems.

Choosing in (46) only the terms whith variables $u_{j}^{i, 0}, j=1,2, \ldots, n$, we receive the expression

$$
\left(\delta+\delta^{2}+\cdots+\delta^{H}\right) \varepsilon \beta^{0}=\delta \sum_{t=0}^{H-1} \delta^{t} \sum_{j=1}^{n} \sum_{i=1}^{m} b_{j}^{i} \sqrt{u_{j}^{i, 0}} .
$$

Maximizing (48) with the constraint (47) by the Lagrange multipliers method, we receive the relations

$$
\frac{b_{j_{2}}^{i}}{b_{j_{1}}^{i}}=\sqrt{\frac{u_{j_{2}}^{i, 0}}{u_{j_{1}}^{i, 0}}}
$$

for any agents $j_{1}$ and $j_{2}$ impacted by the $i$-th firm. Denote as earlier the sum $\sum_{j=1}^{n} u_{j}^{i, 0}$ by $R_{i}^{0}$, we receive

$$
u_{j}^{i, 0}=\frac{\left(b_{j}^{i}\right)^{2} R_{i}^{0}}{\sum_{j=1}^{n}\left(b_{j}^{i}\right)^{2}} .
$$


Substitution of (49) into (48) gives

$$
\delta\left(\sum_{t=0}^{H-1} \delta^{t}\right) \sum_{i=1}^{m} \sqrt{R_{i}^{0} \sum_{j=1}^{n}\left(b_{j}^{i}\right)^{2}} .
$$

Maximizing (50) by $R_{i}^{0}$ with constraint (47), we receive

$$
\left(R_{i}^{0}\right)_{\max }=r_{i}^{0} .
$$

Thus we have

$$
u_{j}^{i, 0}=\frac{\left(b_{j}^{i}\right)^{2}}{\sum_{j=1}^{n}\left(b_{j}^{i}\right)^{2}} r_{i}^{0} .
$$

Let us write the strategy of the $i$-th firm in the $H$-period game. For $t=0,1, \ldots, H-1$ the optimal strategy of the $i$-th firm is

$$
u_{j}^{i, t}=\frac{\left(b_{j}^{i}\right)^{2}}{\sum_{j=1}^{n}\left(b_{j}^{i}\right)^{2}} r_{i}^{t} .
$$

If $t=H$ then $u_{j}^{i, H}=0, j=1,2, \ldots, n$.

Then the principal's problem is the same as the previous problem already solved in searching the numerator on (43), and the denominator of (43) is also expressed by the formula (38). Therefore, in this problem the players' interests are ideally compatible, and $S C I=1$.

Proposition 3. In the model defined in subsection 2.1.

$$
S C I=1 .
$$

Remark 1. A non-trivial situation arises. In the $t$-th period the firm should choose the advertisement cost equal to $\frac{\delta^{2}}{4} \sum_{j=1}^{n}\left(b_{j}^{i}\right)^{2}\left(\sum_{s=0}^{H-1-t} \delta^{s}\right)^{2}$ if this value does not exceed $r_{i}^{t}$, and choose $r_{i}^{t}$ if the value $\frac{\delta^{2}}{4} \sum_{j=1}^{n}\left(b_{j}^{i}\right)^{2}\left(\sum_{s=0}^{H-1-t} \delta^{s}\right)^{2}$ is greater than $r_{i}^{t}$. But the firms do not know the index $H$, and therefore, the value $\frac{\delta^{2}}{4} \sum_{j=1}^{n}\left(b_{j}^{i}\right)^{2}\left(\sum_{s=0}^{H-1-t} \delta^{s}\right)^{2}$. However, they know that the principal never allocates them more resources than $\frac{\delta^{2}}{4} \sum_{j=1}^{n}\left(b_{j}^{i}\right)^{2}\left(\sum_{s=0}^{H-1-t} \delta^{s}\right)^{2}$, and respectively they always choose $r_{i}^{t}$. The principal could use it and allocate to all firms in the $t$-th period more resources than $\frac{\delta^{2}}{4} \sum_{j=1}^{n}\left(b_{j}^{i}\right)^{2}\left(\sum_{s=0}^{H-1-t} \delta^{s}\right)^{2}$, and respectively compel them to invest in the advertisement more than it is advantageous to them. However, it is not advantageous to the principal herself to allocate more than $\frac{\delta^{2}}{4} \sum_{j=1}^{n}\left(b_{j}^{i}\right)^{2}\left(\sum_{s=0}^{H-1-t} \delta^{s}\right)^{2}$ ! Therefore, the principal always allocates the value $\frac{\delta^{2}}{4} \sum_{j=1}^{n}\left(b_{j}^{i}\right)^{2}\left(\sum_{s=0}^{H-1-t} \delta^{s}\right)^{2}$ if it does not exceed her marketing budget $R$, otherwise she allocates the resources as it is optimal for herself. This remains valid until the instant $H-1$ when the principal allocates a reduced amount of resources which provides in the instant $H$ that the sum of values of the state variables is strictly equal to $x^{*}$. Thus, the interests of the principal and the agents (firms) are completely compatible. 


\section{Difference Stackelberg Game with a Constraint on the Sum of State Variables. Case of the Stochastic from the Left Matrix $A$}

Now take a standard assumption that the influence matrix $A$ is stochastic from the left, i.e.

$$
\sum_{j=1}^{n} a_{i j}=1, j=1,2, \ldots, n,
$$

and other assumptions are the same as in the previous section. The model takes the form

$$
\begin{gathered}
J_{0}=\sum_{t=0}^{T} \delta^{t}\left[\sum_{j=1}^{n} z_{j} x_{j}^{t}-\sum_{i=1}^{m} r_{i}^{t}\right] \rightarrow \max , \\
r_{i}^{t} \geq 0, \quad \sum_{i=1}^{m} r_{i}^{t} \leq R, \quad t=0,1,2, \ldots, T, \quad i=1,2, \ldots, m, \\
x_{*} \leq \sum_{j=1}^{n} z_{j} x_{j}^{t} \leq x^{*}, \quad t=0,1,2, \ldots, T-1, T, \\
J_{i}=\sum_{t=0}^{T} \delta^{t}\left[\begin{array}{ll}
\left.\sum_{j=1}^{n}\left(z_{j} x_{j}^{t}-s_{j}^{i} u_{j}^{i, t}\right)\right] & \\
\sum_{j=1}^{n} u_{j}^{i, t} \leq r_{i}^{t}, \quad u_{j}^{i, t} \geq 0, \quad t=0,1,2, \ldots, T, \quad i=1,2, \ldots, m, \\
x_{j}^{t+1}=\sum_{i=1}^{m} b_{j}^{i} \sqrt{u_{j}^{i, t}}+\sum_{l=1}^{n} a_{l j} x_{l}^{t}, \quad x_{j}^{0}=x_{j 0}, \quad j=1,2, \ldots, n,
\end{array}\right. \\
s_{j}^{i}= \begin{cases}1, & \text { if } \quad b_{j}^{i}>0, \\
0, & \text { if } \quad b_{j}^{i}=0,\end{cases}
\end{gathered}
$$

where $z_{j}>0, j=1,2, \ldots, n$, are components of the positive (right) eigenvector which corresponds to the Frobenius eigenvalue 1 of the matrix $A$.

Let us reduce the standard situation to the already considered case. Let $Z$ be an eigenvector corresponding to the eigenvalue 1 of the matrix $A$ :

$$
Z=\left(\begin{array}{c}
z_{1} \\
z_{2} \\
\ldots \\
z_{n}
\end{array}\right), z_{j}>0, j=1,2, \ldots, n .
$$

It is evident that the diagonal matrix

$$
\Xi=\left(\begin{array}{cccc}
z_{1} & 0 & \ldots & 0 \\
0 & z_{2} & \ldots & 0 \\
\ldots & \ldots & \ldots & \ldots \\
0 & 0 & \ldots & z_{n}
\end{array}\right)
$$

is the matrix of transfer to the stochastic (from the right) matrix $P$ :

$$
P=\Xi^{-1} A \Xi .
$$


Then

$$
A=\Xi P \Xi^{-1},
$$

i.e.

$$
A^{\tau}=\Xi^{-1} P^{\tau} \Xi,
$$

and the problem (51)-(57) can be formulated in other coordinates.

Introduce a family of matrices $B^{i}$ :

$$
B^{i}=\left(\begin{array}{cccc}
b_{1}^{i} & 0 & \ldots & 0 \\
0 & b_{2}^{i} & \ldots & 0 \\
\ldots & \ldots & \ldots & \ldots \\
0 & 0 & \ldots & b_{n}^{i}
\end{array}\right)
$$

and vectors

$$
\sqrt{U^{i, t}}=\left(\begin{array}{c}
\sqrt{u_{1}^{i, t}} \\
\sqrt{u_{2}^{i, t}} \\
\cdots \\
\sqrt{u_{n}^{i, t}}
\end{array}\right), i=1,2, \ldots, m
$$

Then the constraint (56) takes the matrix form

$$
X^{t+1}=\sum_{i=1}^{m} B^{i} \sqrt{U^{i, t}}+\Xi^{-1} P^{\tau} \Xi X^{t},
$$

i.e.

$$
Y^{t+1}=\sum_{i=1}^{m} \tilde{B}^{i} \sqrt{U^{i, t}}+P^{\tau} Y^{t}
$$

where

$$
\begin{gathered}
X^{t}=\left(\begin{array}{c}
x_{1}^{t} \\
x_{2}^{t} \\
\ldots \\
x_{n}^{t}
\end{array}\right), \quad Y^{t}=\left(\begin{array}{c}
y_{1}^{t} \\
y_{2}^{t} \\
\ldots \\
y_{n}^{t}
\end{array}\right)=\Xi X^{t}, \\
\tilde{B}^{i}=\Xi B^{i}=\left(\begin{array}{cccc}
z_{1} b_{1}^{i} & 0 & \ldots & 0 \\
0 & z_{2} b_{2}^{i} & \ldots & 0 \\
\ldots & \ldots & \ldots & \ldots \\
0 & 0 & \ldots & z_{n} b_{n}^{i}
\end{array}\right)=\left(\begin{array}{cccc}
\tilde{b}_{1}^{i} & 0 & \ldots & 0 \\
0 & \tilde{b}_{2}^{i} & \ldots & 0 \\
\ldots & \ldots & \ldots \\
0 & 0 & \ldots & \tilde{b}_{n}^{i}
\end{array}\right),
\end{gathered}
$$

or in the coordinate form:

$$
y_{j}^{t+1}=\sum_{i=1}^{m} \tilde{b}_{j}^{i} \sqrt{u_{j}^{i, t}}+\sum_{l=1}^{n} p_{l j} y_{l}^{t},
$$

and the constraint (53) looks like

$$
x_{*} \leq \sum_{j=1}^{n} y_{j}^{t} \leq x^{*} .
$$


The expression (54) takes the form

$$
J_{i}=\sum_{t=0}^{T} \delta^{t}\left[\sum_{j=1}^{n}\left(y_{j}^{t}-s_{j}^{i} u_{j}^{i, t}\right)\right] \rightarrow \max ,
$$

and (51) takes the form

$$
J_{0}=\sum_{t=0}^{T} \delta^{t}\left[\sum_{j=1}^{n} y_{j}^{t}-\sum_{i=1}^{m} r_{i}^{t}\right] \rightarrow \max .
$$

Other expressions in the problem (51)-(57) do not change. Based on the solution of the problem from the Section 2, we find the optimal strategies of the firms:

$$
u_{j}^{i, t}=\frac{\left(\tilde{b_{j}^{i}}\right)^{2} r_{i}^{t}}{\sum_{j=1}^{n}\left(\tilde{b_{j}^{i}}\right)^{2}}=\frac{\left(z_{j} b_{j}^{i}\right)^{2} r_{i}^{t}}{\sum_{j=1}^{n}\left(z_{j} b_{j}^{i}\right)^{2}}, \quad i=1,2, \ldots, m, \quad t=1,2, \ldots, T .
$$

As for the principal, let in the moment $t=H$ the value

$$
\begin{gathered}
\Delta=\sqrt{\sum_{i=1}^{m} \sum_{j=1}^{n}\left(z_{j} b_{j}^{i}\right)^{2}} \min \left\{\frac{\delta}{2} \sqrt{\sum_{i=1}^{m} \sum_{j=1}^{n}\left(z_{j} b_{j}^{i}\right)^{2}}, \sqrt{R}\right\}+ \\
+\sqrt{\sum_{i=1}^{m} \sum_{j=1}^{n}\left(z_{j} b_{j}^{i}\right)^{2}} \min \left\{\frac{\delta}{2} \sqrt{\sum_{i=1}^{m} \sum_{j=1}^{n}\left(z_{j} b_{j}^{i}\right)^{2}}(1+\delta), \sqrt{R}\right\}+\cdots+ \\
+\sqrt{\sum_{i=1}^{m} \sum_{j=1}^{n}\left(z_{j} b_{j}^{i}\right)^{2}} \min \left\{\frac{\delta}{2} \sqrt{\sum_{i=1}^{m} \sum_{j=1}^{n}\left(z_{j} b_{j}^{i}\right)^{2}}\left(\sum_{s=0}^{H-1-t} \delta^{s}\right), \sqrt{R}\right\}+\cdots+ \\
+\sqrt{\sum_{i=1}^{m} \sum_{j=1}^{n}\left(z_{j} b_{j}^{i}\right)^{2}} \min \left\{\frac{\delta}{2} \sqrt{\sum_{i=1}^{m} \sum_{j=1}^{n}\left(z_{j} b_{j}^{i}\right)^{2}}\left(\sum_{s=0}^{H-1} \delta^{s}\right), \sqrt{R}\right\}
\end{gathered}
$$

for the first time becomes greater or equal than $x^{*}-\sum_{j=1}^{n} z_{j} x_{j}^{0}$ (if it is possible for some $t \leq T)$. Denote

$$
\Delta_{1}=x^{*}-\sum_{j=1}^{n} z_{j} x_{j}^{0}-\left(\Delta-\sqrt{\sum_{i=1}^{m} \sum_{j=1}^{n}\left(z_{j} b_{j}^{i}\right)^{2}} \min \left\{\frac{\delta}{2} \sqrt{\sum_{i=1}^{m} \sum_{j=1}^{n}\left(z_{j} b_{j}^{i}\right)^{2}}, \sqrt{R}\right\}\right),
$$

we find the total amount of resources that the principal should allocate to the firms in the moment $H-1$ so that in the moment $H$ the sum

$$
\sum_{j=1}^{n} z_{j} x_{j}
$$

be strictly equal to $x^{*}$ :

$$
\tilde{R}=\frac{\left(\Delta_{1}\right)^{2}}{\sum_{i=1}^{m} \sum_{j=1}^{n}\left(z_{j} b_{j}^{i}\right)^{2}}
$$


Thus, the principal's optimal strategy is the following:

when $0 \leq t \leq H-2$

$$
r_{i}^{t}=\frac{\sum_{j=1}^{n}\left(z_{j} b_{j}^{i}\right)^{2}}{\sum_{i=1}^{m} \sum_{j=1}^{n}\left(z_{j} b_{j}^{i}\right)^{2}} \min \left\{\frac{\delta^{2}}{4} \sum_{i=1}^{m} \sum_{j=1}^{n}\left(z_{j} b_{j}^{i}\right)^{2}\left(\sum_{s=0}^{H-1-t} \delta^{s}\right)^{2}, R\right\}
$$

when $t=H-1$

when $H \leq t \leq T$

$$
r_{i}^{H-1}=\frac{\sum_{j=1}^{n}\left(z_{j} b_{j}^{i}\right)^{2}}{\sum_{i=1}^{m} \sum_{j=1}^{n}\left(z_{j} b_{j}^{i}\right)^{2}} \tilde{R}
$$

$$
r_{i}^{t}=0, \quad i=1,2, \ldots, m
$$

The maximal guaranteed payoff of the principal is equal to

$$
\begin{aligned}
& \left(\sum_{s=0}^{H-1} \delta^{s}\right) \sum_{j=1}^{n} z_{j} x_{j}^{0}+\left(\sum_{s=1}^{H-1} \delta^{s}\right) \sqrt{R^{0} \sum_{i=1}^{m} \sum_{j=1}^{n}\left(z_{j} b_{j}^{i}\right)^{2}}-R^{0}+ \\
& +\left(\sum_{s=2}^{H-1} \delta^{s}\right) \sqrt{R^{1} \sum_{i=1}^{m} \sum_{j=1}^{n}\left(z_{j} b_{j}^{i}\right)^{2}}-\delta R^{1}+\cdots+ \\
& +\left(\sum_{s=t+1}^{H-1} \delta^{s}\right) \sqrt{R^{t} \sum_{i=1}^{m} \sum_{j=1}^{n}\left(z_{j} b_{j}^{i}\right)^{2}}-\delta^{t} R^{t}+\cdots+ \\
& +\delta^{H-1} \sqrt{R^{H-2} \sum_{i=1}^{m} \sum_{j=1}^{n}\left(z_{j} b_{j}^{i}\right)^{2}-\delta^{H-2} R^{H-2}-\delta^{H-1} \tilde{R}+x^{*} \sum_{h=H}^{T} \delta^{h}}
\end{aligned}
$$

where, as can be seen from (34),

$$
\begin{gathered}
R^{0}=\min \left\{\frac{\delta^{2}}{4} \sum_{i=1}^{m} \sum_{j=1}^{n}\left(z_{j} b_{j}^{i}\right)^{2}\left(\sum_{s=0}^{H-1} \delta^{s}\right)^{2}, R\right\}, \\
R^{1}=\min \left\{\frac{\delta^{2}}{4} \sum_{i=1}^{m} \sum_{j=1}^{n}\left(z_{j} b_{j}^{i}\right)^{2}\left(\sum_{s=0}^{H-2} \delta^{s}\right)^{2}, R\right\}, \ldots, \\
R^{t}=\min \left\{\frac{\delta^{2}}{4} \sum_{i=1}^{m} \sum_{j=1}^{n}\left(z_{j} b_{j}^{i}\right)^{2}\left(\sum_{s=0}^{H-1-t} \delta^{s}\right)^{2}, R\right\}, \ldots, \\
R^{H-2}=\min \left\{\frac{\delta^{2}}{4} \sum_{i=1}^{m} \sum_{j=1}^{n}\left(z_{j} b_{j}^{i}\right)^{2}(1+\delta)^{2}, R\right\},
\end{gathered}
$$

where $R^{t}$ denotes the sum $\sum_{i=1}^{m} r_{i}^{t}, t=0,1,2, \ldots, H-2$, and the value $\tilde{R}$ is determined by the expression (58).

It is clear that $S C I=1$ as in the previous problem from the Section 2. The expressions for the maximal guaranteed payoff in this case differ from those in the section 2 only by the multipliers $z_{j}$ - the components of Frobenius eigenvector of matrix $A$. So, in this problem with the homeostasis condition, as in the similar problem without this condition, the interests of players are completely compatible. 
Game Theoretic Models of Sustainable Management in Marketing Networks

\section{Differential Stackelberg Game with a Constraint on the Sum of State Variables. Case of the Stochastic (from the Right) Matrix $A$}

\subsection{The Problem Statement}

Consider this problem in continuous time. Now the model takes the form

$$
\begin{gathered}
J_{0}=\int_{0}^{T} e^{-\rho t}\left[\sum_{j=1}^{n} x_{j}(t)-\sum_{i=1}^{m} r_{i}(t)\right] d t \rightarrow \max , \\
r_{i}(t) \geq 0, \quad \sum_{i=1}^{m} r_{i}(t) \leq R, \quad t \in[0, T], \quad i=1,2, \ldots, m, \\
x_{*} \leq \sum_{j=1}^{n} x_{j}(t) \leq x^{*}, \quad t \in[0, T], \\
J_{i}=\int_{0}^{T} e^{-\rho t}\left[\sum_{j=1}^{n}\left(x_{j}(t)-s_{j}^{i} u_{j}^{i}(t)\right)\right] d t \rightarrow \max , \\
\sum_{j=1}^{n} u_{j}^{i}(t) \leq r_{i}(t), \quad u_{j}^{i}(t) \geq 0, \quad t \in[0, T], \quad i=1,2, \ldots, m, \\
\dot{x}_{j}=\sum_{i=1}^{m} b_{j}^{i} \sqrt{u_{j}^{i}(t)}+\sum_{l=1}^{n} \tilde{a}_{l j} x_{l}(t), \quad x_{j}(0)=x_{j 0}, \quad j=1,2, \ldots, n, \\
s_{j}^{i}= \begin{cases}1, & \text { if } \quad b_{j}^{i}>0, \\
0, & \text { if } \quad b_{j}^{i}=0 .\end{cases}
\end{gathered}
$$

Here $a_{i j}$ is a coefficient of influence of the $i$-th basic agent to the $j$-th basic agent in the discrete model. When we move from the discrete description to the continuous one, the influence matrix $A$ is substituted by the matrix $\tilde{A}$ in the form

$$
\tilde{A}=A-I \text {. }
$$

Thus,

$$
\tilde{a}_{i j}=\left\{\begin{array}{c}
a_{i j}, \text { if } \quad i \neq j, \\
a_{i j}-1, \quad \text { if } \quad i=j .
\end{array}\right.
$$

Let us first assume that the matrix $A$ is stochastic (from the right), i.e.

$$
\sum_{j=1}^{n} a_{i j}=1, \quad i=1,2, \ldots, n .
$$

Solving the problem of the $i$-th firm, we can substitute $n$ state variables $x_{j}$ by their sum and denote by $x$ the only state variable:

$$
\sum_{j=1}^{n} x_{j}=x, \quad \sum_{j=1}^{n} x_{j 0}=x_{0},
$$


then

$$
\sum_{j=1}^{n} \sum_{l=1}^{n} \tilde{a}_{l j} x_{l}=\sum_{j=1}^{n} \sum_{l=1}^{n} a_{l j} x_{l}-\sum_{j=1}^{n} x_{j}=\sum_{l=1}^{n} x_{l}-\sum_{j=1}^{n} x_{j}=x-x=0,
$$

and the conditions (62) and (64) respectively take the form

$$
J_{i}=\int_{0}^{T} e^{-\rho t}\left[x-\sum_{j=1}^{n} s_{j}^{i} u_{j}^{i}(t)\right] d t \rightarrow \max ,
$$

and

$$
\dot{x}=\sum_{j=1}^{n} \sum_{i=1}^{m} b_{j}^{i} \sqrt{u_{j}^{i}(t)}, \quad x(0)=x_{0} .
$$

\subsection{Solution the Problem of $\boldsymbol{i}$-th Firm}

The Hamilton-Jacobi-Bellman equation has the form

$$
\rho V_{i}-\frac{\partial V_{i}}{\partial t}=\max _{u_{j}^{i}, 1 \leq j \leq n}\left\{x(t)-\sum_{j=1}^{n} s_{j}^{i} u_{j}^{i}(t)+\frac{\partial V_{i}}{\partial x} \sum_{j=1}^{n} \sum_{k=1}^{m} b_{j}^{k} \sqrt{u_{j}^{k}(t)}\right\}
$$

with constraint

$$
\sum_{j=1}^{n} u_{j}^{i}(t) \leq r_{i}(t)
$$

Maximizing by $u_{j}^{i}, j=1,2, \ldots, n, b_{j}^{i} \neq 0$, we receive

$$
\frac{\partial V_{i}}{\partial x} b_{j}^{i} \frac{1}{2}\left(u_{j}^{i}\right)^{-\frac{1}{2}}-1=\mu
$$

where $\mu$ is a Lagrange multiplier. Then for any $1 \leq j_{1}, j_{2} \leq n$ :

$$
\frac{\left(\partial V_{i} / \partial x\right) b_{j_{2}}^{i}}{\left(\partial V_{i} / \partial x\right) b_{j_{1}}^{i}}=\frac{b_{j_{2}}^{i}}{b_{j_{1}}^{i}}=\left(\frac{u_{j_{2}}^{i}}{u_{j_{1}}^{i}}\right)^{\frac{1}{2}} .
$$

Denote the sum

$$
\frac{u_{j_{1}}^{i}}{\left(b_{j_{1}}^{i}\right)^{2}} \sum_{j=1}^{n}\left(b_{j}^{i}\right)^{2}
$$

by $R_{i}(t)$. Then

$$
u_{j}^{i}=\frac{R_{i}(t)\left(b_{j}^{i}\right)^{2}}{\sum_{j=1}^{n}\left(b_{j}^{i}\right)^{2}} .
$$

As we take the linear Bellman functions,

$$
V_{i}(x, t)=\alpha^{i}(t) x+\beta^{i}(t),
$$

then we can write the equation (68) with consideration of (69) in the form

$$
\rho \alpha^{i}(t) x+\rho \beta^{i}(t)-\alpha^{\prime i}(t) x-\beta^{\prime i}(t)=x-R_{i}(t)+\alpha^{i}(t) \sum_{k=1}^{m} \sum_{j=1}^{n} \frac{b_{j}^{k} \sqrt{R_{k}(t)} b_{j}^{k}}{\sqrt{\sum_{j=1}^{n}\left(b_{j}^{k}\right)^{2}}},
$$


or

$$
\rho \alpha^{i}(t) x+\rho \beta^{i}(t)-\alpha^{\prime i}(t) x-\beta^{\prime i}(t)=x-R_{i}(t)+\alpha^{i}(t) \sum_{k=1}^{m} \sqrt{R_{k}(t) \sum_{j=1}^{n}\left(b_{j}^{k}\right)^{2}} .
$$

Equation of the coefficients at the variable $x$ in the left and right hand sides of the equation (26), we receive a differential equation for $\alpha^{i}(t)$. Its solution gives

$$
\alpha^{i}(t)=C e^{\rho t}+\frac{1}{\rho} .
$$

Using the boundary condition $\alpha^{i}(T)=0$, we find the integration constant

$$
C=-\frac{1}{\rho} e^{\rho T} .
$$

Therefore,

$$
\alpha^{i}(t)=\frac{1}{\rho}\left(1-e^{\rho(t-T)}\right) .
$$

Function $\alpha^{i}(t)$ is the same for all firms, then we will omit the index $i$ of the functions $\alpha^{i}(t)$. Equating the constant terms in the left and right hand sides of the equation (26), we receive a differential equation for $\beta^{i}(t)$ :

$$
\beta^{\prime}(t)-\rho \beta^{i}(t)=R_{i}(t)-\alpha^{i}(t) \sum_{k=1}^{m} \sqrt{R_{k}(t) \sum_{j=1}^{n}\left(b_{j}^{k}\right)^{2}},
$$

and solve it by the method of variation of parameters. We have:

$$
\begin{gathered}
\beta^{i}(t)=e^{\rho t} C(t) \\
C^{\prime}(t) e^{\rho t}=R_{i}(t)-\alpha(t) \sum_{k=1}^{m} \sqrt{R_{k}(t) \sum_{j=1}^{n}\left(b_{j}^{k}\right)^{2}}, \\
C(t)=\int_{0}^{t} e^{-\rho \tau}\left[R_{i}(\tau)-\alpha(\tau) \sum_{k=1}^{m} \sqrt{R_{k}(\tau) \sum_{j=1}^{n}\left(b_{j}^{k}\right)^{2}}\right] d \tau+C .
\end{gathered}
$$

Considerating the boundary condition $\beta^{i}(T)=0$, we receive

$$
C=-\int_{0}^{t} e^{-\rho \tau}\left[R_{i}(\tau)-\alpha(\tau) \sum_{k=1}^{m} \sqrt{R_{k}(\tau) \sum_{j=1}^{n}\left(b_{j}^{k}\right)^{2}}\right] d \tau
$$

Therefore,

$$
\beta^{i}(t)=e^{\rho t} \int_{t}^{T} e^{-\rho \tau}\left[\alpha(\tau) \sum_{k=1}^{m} \sqrt{R_{k}(\tau) \sum_{j=1}^{n}\left(b_{j}^{k}\right)^{2}}-R_{i}(\tau)\right] d \tau
$$


Choosing the maximal value of the right hand side of the expression (26) in dependence of $R_{i}(t)$, we have

$$
-R_{i}(t)+\alpha^{i}(t) \sqrt{R_{i}(t)} \sqrt{\sum_{j=1}^{n}\left(b_{j}^{i}\right)^{2}} \rightarrow \max ,
$$

hence

$$
\left(R_{i}(t)\right)_{\max }=\frac{1}{4}(\alpha(t))^{2} \sum_{j=1}^{n}\left(b_{j}^{i}\right)^{2} .
$$

Thus, the value $R_{i}(t)$ (omitting the index $i$ at $\alpha^{i}(t)$ ) is equal to

$$
R_{i}(t)=\min \left\{\frac{1}{4}(\alpha(t))^{2} \sum_{j=1}^{n}\left(b_{j}^{i}\right)^{2}, r_{i}(t)\right\} .
$$

Thus we have proved the statement.

Proposition 4. The optimal strategies of firms in problem (66)-(67) are defined by expression (69), where $R_{i}(t)$ defined by (27) and

$$
\alpha(t)=\frac{1}{\rho}\left(1-e^{\rho(t-T)}\right) .
$$

\subsection{The Principal's Problem}

Now consider the principal's strategy. The principal cannot decrease the value $\sum_{j=1}^{n} x_{j}$; she can only increase it by allocating the resources to firms. Then her optimal strategy is evident. From the problem formulation it follows that $\sum_{j=1}^{n} x_{j 0} \geq$ $x_{*}$. The principal solves the problem:

$$
J_{0}=\int_{0}^{T} e^{-\rho t}\left(\sum_{j=1}^{n} x_{j}(t)-\sum_{i=1}^{m} r_{i}(t)\right) d t \rightarrow \max ,
$$

with constraints $(60)-(61),(63)-(65)$.

It is clear that for maximization of her payoff the principal should increase the value $\sum_{j=1}^{n} x_{j}(t)$ as in the previous discrete problem but only up to the value $x^{*}$, after what she must cease to allocate resources to the firms. Then the value $\sum_{j=1}^{n} x_{j}(t)$ will remain equal to $x^{*}$ until the end of the game. For implementation of this strategy the principal should already at the instant $t=0$ determine the instant $t=h$ when the value $\sum_{j=1}^{n} x_{j}(t)$ becomes equal to $x^{*}$ given the respective strategy.

As according to (27) the principal will never allocate to any firm $i$ in any instant $t$ more resources than

$$
\frac{1}{4}(\alpha(t))^{2} \sum_{j=1}^{n}\left(b_{j}^{i}\right)^{2},
$$

we can rewrite the expression (69) in the form

$$
u_{j}^{i}=\frac{r_{i}(t)\left(b_{j}^{i}\right)^{2}}{\sum_{j=1}^{n}\left(b_{j}^{i}\right)^{2}} .
$$


Substituting to the equation (67) for the sum of variables $x$ the expression (72), we receive

$$
\dot{x}=\sum_{i=1}^{m} \sqrt{r_{i}(t) \sum_{j=1}^{n}\left(b_{j}^{i}\right)^{2}}, \quad x(0)=x_{0} .
$$

Integration the equation (73), we find

$$
x(t)=\int_{0}^{t} \sum_{i=1}^{m} \sqrt{r_{i}(\tau) \sum_{j=1}^{n}\left(b_{j}^{i}\right)^{2}} d \tau+x_{0}
$$

Thus, $h=x^{-1}\left(x^{*}\right)$ is the instant of time when the right hand side of the expression (74) becomes equal to $x^{*}$.

Given the value of $h$ and using the assumption about stochasticity of the influence matrix, we can reformulate the principal's problem as follows:

$$
J_{0}=\int_{0}^{h} e^{-\rho t}\left(x(t)-\sum_{i=1}^{m} r_{i}(t)\right) d t \rightarrow \max ,
$$

with constraints

$$
r_{i}(t) \geq 0, \quad \sum_{i=1}^{m} r_{i}(t) \leq R, \quad t \in[0, h], \quad i=1,2, \ldots, m
$$

and (73).

The Hamilton-Jacobi-Bellman equation takes the form:

$$
\rho V_{0}-\frac{\partial V_{0}}{\partial t}=\max _{r_{i}, 1 \leq i \leq m}\left\{x(t)-\sum_{i=1}^{m} r_{i}(t)+\frac{\partial V_{0}}{\partial x} \sum_{i=1}^{m} \sqrt{r_{i}(t) \sum_{j=1}^{n}\left(b_{j}^{i}\right)^{2}}\right\}
$$

with constrain

$$
\sum_{i=1}^{m} r_{i}(t) \leq R .
$$

We take the linear Bellman function:

$$
V_{0}(x, t)=\alpha^{0}(t) x+\beta^{0}(t) .
$$

Substituting (77) into (76), we receive

$$
\begin{gathered}
\rho \alpha^{0}(t) x+\rho \beta^{0}(t)-\alpha^{\prime 0}(t) x-\beta^{\prime 0}(t)= \\
=\max _{r_{i}(t), 1 \leq i \leq m}\left\{x(t)-\sum_{i=1}^{m} r_{i}(t)+\alpha^{0}(t) \sum_{i=1}^{m} \sqrt{r_{i}(t) \sum_{j=1}^{n}\left(b_{j}^{i}\right)^{2}}\right\} .
\end{gathered}
$$

Equating in the left and right hand sides of the equation (78) the coefficients at the variable $x$, we receive a differential equation for $\alpha^{0}(t)$ :

$$
\alpha^{\prime}(t)-\rho \alpha^{0}(t)=-1 .
$$


It coincides with the differential equations for $\alpha^{i}(t)$ but has other boundary condition

$$
\alpha^{0}(h)=0 .
$$

Therefore,

$$
\alpha^{0}(t)=\frac{1}{\rho}\left(1-e^{\rho(t-h)}\right), \quad t \in[0, h] .
$$

Equating the constant terms in the left and right hand sides of the equation (78), we receive a differential equation for $\beta^{0}(t)$ :

$$
\beta^{\prime 0}(t)-\rho \beta^{0}(t)=\sum_{i=1}^{m} r_{i}(t)-\alpha^{0}(t) \sum_{i=1}^{m} \sqrt{r_{i}(t) \sum_{j=1}^{n}\left(b_{j}^{i}\right)^{2}} .
$$

Its solution by the method of variation of parameters gives

$$
\begin{gathered}
\beta^{0}(t)=C(t) e^{\rho t} \\
C^{\prime}(t) e^{\rho t}=\sum_{i=1}^{m} r_{i}(t)-\alpha^{0}(t) \sum_{i=1}^{m} \sqrt{r_{i}(t) \sum_{j=1}^{n}\left(b_{j}^{i}\right)^{2}} \\
C(t)=\int_{0}^{t}\left(\sum_{i=1}^{m} r_{i}(\tau)-\alpha^{0}(\tau) \sum_{i=1}^{m} \sqrt{r_{i}(\tau) \sum_{j=1}^{n}\left(b_{j}^{i}\right)^{2}}\right) e^{-\rho \tau} d \tau+C .
\end{gathered}
$$

Using the boundary condition

$$
\beta^{0}(h)=0,
$$

we find

$$
C=-\int_{0}^{t}\left(\sum_{i=1}^{m} r_{i}(\tau)-\alpha^{0}(\tau) \sum_{i=1}^{m} \sqrt{r_{i}(\tau) \sum_{j=1}^{n}\left(b_{j}^{i}\right)^{2}}\right) e^{-\rho \tau} d \tau .
$$

Therefore,

$$
\begin{gathered}
C(t)=\int_{t}^{h}\left(\alpha^{0}(\tau) \sum_{i=1}^{m} \sqrt{r_{i}(\tau) \sum_{j=1}^{n}\left(b_{j}^{i}\right)^{2}}-\sum_{i=1}^{m} r_{i}(\tau)\right) e^{-\rho \tau} d \tau \\
\beta^{0}(t)=e^{\rho t} \int_{t}^{h}\left[\alpha^{0}(\tau)\left(\sum_{i=1}^{m} \sqrt{r_{i}(\tau) \sum_{j=1}^{n}\left(b_{j}^{i}\right)^{2}}\right)-\sum_{i=1}^{m} r_{i}(\tau)\right] e^{-\rho \tau} d \tau .
\end{gathered}
$$

Particularly, when $t=0$ we have

$$
\beta^{0}(0)=\int_{0}^{h}\left[\alpha^{0}(\tau)\left(\sum_{i=1}^{m} \sqrt{r_{i}(\tau) \sum_{j=1}^{n}\left(b_{j}^{i}\right)^{2}}\right)-\sum_{i=1}^{m} r_{i}(\tau)\right] e^{-\rho \tau} d \tau .
$$

Maximizing the right hand side of the expression (78) by $r_{i}(t), i=1,2, \ldots, m$, with constraint $\sum_{i=1}^{m} r_{i}(t) \leq R$, we receive

$$
1-\alpha^{0}(t) \frac{\sqrt{\sum_{j=1}^{n}\left(b_{j}^{i}\right)^{2}}}{2 \sqrt{r_{i}(t)}}=\mu,
$$


where $\mu$ is a Lagrange multiplier. Then

$$
\frac{\sqrt{\sum_{j=1}^{n}\left(b_{j}^{i_{1}}\right)^{2}}}{\sqrt{\sum_{j=1}^{n}\left(b_{j}^{i_{2}}\right)^{2}}}=\frac{\sqrt{r_{i_{1}}(t)}}{\sqrt{r_{i_{2}}(t)}} .
$$

Therefore, for any $1 \leq i_{1}, i_{2} \leq m$ it is true that

$$
r_{i_{2}}(t)=\frac{\sum_{j=1}^{n}\left(b_{j}^{i_{2}}\right)^{2}}{\sum_{j=1}^{n}\left(b_{j}^{i_{1}}\right)^{2}} r_{i_{1}}(t) .
$$

Denote the sum $\sum_{i=1}^{m} r_{i}(t)$ by $r(t)$. Then

$$
r_{i_{1}}(t) \sum_{i=1}^{m} \sum_{j=1}^{n}\left(b_{j}^{i}\right)^{2}=r(t) \sum_{j=1}^{n}\left(b_{j}^{i_{1}}\right)^{2} .
$$

Therefore,

$$
r_{i}(t)=r(t) \frac{\sum_{j=1}^{n}\left(b_{j}^{i}\right)^{2}}{\sum_{k=1}^{m} \sum_{j=1}^{n}\left(b_{j}^{k}\right)^{2}} .
$$

Substituting (81) in the right hand side of (78) we receive:

$$
x(t)-r(t)+\alpha^{0}(t) \sqrt{r(t) \sum_{i=1}^{m} \sum_{j=1}^{n}\left(b_{j}^{i}\right)^{2}} .
$$

A non-conditional optimization of this expression by $r(t)$ gives

$$
(r(t))_{\text {max }}=\frac{1}{4}\left(\alpha^{0}(t)\right)^{2} \sum_{i=1}^{m} \sum_{j=1}^{n}\left(b_{j}^{i}\right)^{2} .
$$

Thus, for the instants $t \in[0, h]$ when

$$
R \geq \frac{1}{4}\left(\alpha^{0}(t)\right)^{2} \sum_{i=1}^{m} \sum_{j=1}^{n}\left(b_{j}^{i}\right)^{2},
$$

the principal's optimal strategy is

$$
r_{i}(t)=\frac{1}{4}\left(\alpha^{0}(t)\right)^{2} \sum_{j=1}^{n}\left(b_{j}^{i}\right)^{2}, \quad i=1,2, \ldots, m .
$$

For the instants $t \in[0, h]$ when

$$
R<\frac{1}{4}\left(\alpha^{0}(t)\right)^{2} \sum_{i=1}^{m} \sum_{j=1}^{n}\left(b_{j}^{i}\right)^{2},
$$

the principal's optimal strategy is

$$
r_{i}(t)=\frac{\sum_{j=1}^{n}\left(b_{j}^{i}\right)^{2}}{\sum_{k=1}^{m} \sum_{j=1}^{n}\left(b_{j}^{k}\right)^{2}} R \quad i=1,2, \ldots, m .
$$

Combining the two expressions for $r_{i}(t)$ in the one formula, we receive following statement. 
Proposition 5. The principal's optimal control is

$$
\begin{gathered}
r_{i}(t)=\frac{\sum_{j=1}^{n}\left(b_{j}^{i}\right)^{2}}{\sum_{k=1}^{m} \sum_{j=1}^{n}\left(b_{j}^{k}\right)^{2}} \min \left\{\frac{1}{4}\left(\alpha^{0}(t)\right)^{2} \sum_{k=1}^{m} \sum_{j=1}^{n}\left(b_{j}^{k}\right)^{2}, R\right\}, \quad t \in[0, h], \\
r_{i}(t)=0, \quad t \in[h, T], \quad i=1,2, \ldots, m,
\end{gathered}
$$

where

$$
\alpha^{0}(t)=\frac{1}{\rho}\left(1-e^{\rho(t-h)}\right) .
$$

Using (79) and (80), we can write:

$$
\begin{gathered}
\max _{r_{i}, 1 \leq i \leq m} \hat{J}_{0}=V_{0}(x(0), 0)=\alpha^{0}(0) x(0)+\beta^{0}(0)= \\
=\frac{1}{\rho}\left(1-e^{-\rho h}\right) x_{0}+\int_{0}^{h}\left[\alpha^{0}(\tau)\left(\sum_{i=1}^{m} \sqrt{r_{i}(\tau) \sum_{j=1}^{n}\left(b_{j}^{i}\right)^{2}}\right)-\sum_{i=1}^{m} r_{i}(\tau)\right] e^{-\rho \tau} d \tau,
\end{gathered}
$$

when the value $r_{i}(\tau)$ is determined by the expression $(82)$, and $\alpha^{0}(\tau)$ - by the expression (79). Then according to (83) the principal's guaranteed payoff is equal to

$$
\begin{gathered}
\max _{r_{i}, 1 \leq i \leq m} J_{0}+x^{*} \int_{h}^{T} e^{-\rho t} d t=\frac{1}{\rho}\left(e^{-\rho h}-e^{-\rho T}\right) x^{*}+\frac{1}{\rho}\left(1-e^{-\rho h}\right) x_{0}+ \\
+\int_{0}^{h}\left[\alpha^{0}(\tau)\left(\sum_{i=1}^{m} \sqrt{r_{i}(\tau) \sum_{j=1}^{n}\left(b_{j}^{i}\right)^{2}}\right)-\sum_{i=1}^{m} r_{i}(\tau)\right] e^{-\rho \tau} d \tau,
\end{gathered}
$$

where the instant $h$ is determined by the expression (74).

\subsection{System Compatibility Index}

Calculate the system compatibility index:

$$
S C I=\frac{\max _{\left\{r_{i}\right\}_{i=1}^{m}} \min _{\left\{u_{j}^{i}\right\}_{i=1 ; j=1}^{m ;} \in N E\left(\left\{r_{i}\right\}_{i=1}^{m}\right)} J_{0}\left(\left\{r_{i}\right\}_{i=1}^{m},\left\{u_{j}^{i}\right\}_{i=1 ; j=1}^{m ;} \quad n\right)}{\max _{\left\{r_{i}\right\}_{i=1}^{m}} \max _{\left\{u_{j}^{i}\right\}_{i=1 ; j=1}^{m ;}} J_{0}\left(\left\{r_{i}\right\}_{i=1}^{m},\left\{u_{j}^{i}\right\}_{i=1 ; j=1}^{m ;}\right)} .
$$

As soon, SCI shows the measure of compatability of system. The numerator of (85) is the payoff of the principal in the case if the firms choose the most unfavorable for principal equilibrium strategies. The denumerator of (85) is the payoff of the principal in the case if the firms choose the most favorable for principal strategies.

Given the principal's strategy the set of equilibrium strategies of each agent is one-element in our case, so the numerator of the expression (85) is equal to the right hand side of (84). For calculation of the denominator in the expression (85) let us assume that each agent maximizes not his payoff but the principal's payoff given her strategy, i.e. the agent solves the problem

$$
\tilde{J}_{i}=\int_{0}^{T} e^{-\rho t} \sum_{j=1}^{n} x_{j}(t) d t \rightarrow \max
$$

with constraints (61) and (63) - (65). 
If $\sum_{j=1}^{n} x_{j}^{0} \geq x^{*}$ then the optimal strategy of each firm is evident: it does not invest to the advertisement. Moreover, it is impossible because in this case the principal does not allocate any financial resource to the firms, i.e. then $u_{j}^{i, t}=0$.

Let $\sum_{j=1}^{n} x_{j}^{0}<x^{*}$. Unlike the principal, any agent is unable to estimate a priori in which instant of time $h$ the sum of values of the state variables becomes equal to $x^{*}$, but it is redundant because the optimal strategies of the firms do not depend on the game's length.

Consider the game in the time segment $[0, h]$. Similar to the Section 3 we receive for the optimal strategies of all firms the expressions similar to (69), (27), (79):

$u_{j}^{i}(t)=\frac{R_{i}(t)\left(b_{j}^{i}\right)^{2}}{\sum_{j=1}^{n}\left(b_{j}^{i}\right)^{2}}, R_{i}(t)=\min \left\{\frac{1}{4} \alpha(t) \sum_{j=1}^{n}\left(b_{j}^{i}\right)^{2}, r_{i}(t)\right\}, \alpha(t)=\frac{1}{\rho}\left(1-e^{\rho(t-h)}\right)$.

As the principal will never allocate to any firm more resources than

$$
\frac{1}{4} \alpha(t) \sum_{j=1}^{n}\left(b_{j}^{i}\right)^{2}
$$

we can write

$$
u_{j}^{i}(t)=\frac{\left(b_{j}^{i}\right)^{2} r_{i}(t)}{\sum_{j=1}^{n}\left(b_{j}^{i}\right)^{2}}, \quad i=1,2, \ldots, m, \quad j=1,2, \ldots, n, \quad t \in[0, h] .
$$

Then the principal's problem is the same as already solved, and the denominator in (85) is also expressed by the formula (84). Therefore, in the problem with constraint on the sum of state variables the interests of players are completely compatible, i.e. $S C I=1$.

Proposition 6. In the problem defined in subsection 4.1.

$$
S C I=1 .
$$

Remark 2. A non-trivial situation arises again. At the instant $t$ the firms should choose the advertisement cost equal to $(1 / 4 \rho)\left(1-e^{\rho(t-h)}\right) \sum_{j=1}^{n}\left(b_{j}^{i}\right)^{2}$ if this value does not exceed $r_{i}^{t}$, and choose $r_{i}^{t}$, otherwise. But the firms do not know the instant $h$, and the value $(1 / 4 \rho)\left(1-e^{\rho(t-h)}\right) \sum_{j=1}^{n}\left(b_{j}^{i}\right)^{2}$, respectively. However, they know that the principal will never allocate to them more resources than $(1 / 4 \rho)\left(1-e^{\rho(t-h)}\right) \sum_{j=1}^{n}\left(b_{j}^{i}\right)^{2}$, and they always choose $r_{i}^{t}$. The principal could use it and allocate to all firms in the moment $t$ more resources than $(1 / 4 \rho)\left(1-e^{\rho(t-h)}\right) \sum_{j=1}^{n}\left(b_{j}^{i}\right)^{2}$, and compel them to invest more. However, it is not advantageous for the principal herself to allocate more than $(1 / 4 \rho)\left(1-e^{\rho(t-h)}\right) \sum_{j=1}^{n}\left(b_{j}^{i}\right)^{2}$. Therefore, she always allocates $(1 / 4 \rho)\left(1-e^{\rho(t-h)}\right) \sum_{j=1}^{n}\left(b_{j}^{i}\right)^{2}$, if this value does not exceed her marketing budget $R$, otherwise she allocates the resources in the way optimal to her. This strategy remains valid until the instant $h$, after what the principal does not allocate more resources because the value $x^{*}$ is achieved optimally. Thus, in the problem with constraint on the sum of state variables the interests of the principal and the firms are completely coordinated as in the problem without the homeostasis condition. 


\section{Differential Stackelberg Game with a Constraint on the Sum of State Variables. Case of the Stochastic from the Left Matrix $A$}

Now take a standard assumption that the influence matrix $A$ is stochastic from the left, i.e.

$$
\sum_{j=1}^{n} a_{i j}=1, j=1,2, \ldots, n
$$

Let $z_{j}>0, j=1,2, \ldots, n$ are components of the positive (right) eigenvector corresponding to the Frobenius eigenvalue 1 of the matrix $A$.

The model takes the form

$$
\begin{gathered}
J_{0}=\int_{t=0}^{T} e^{-\rho t}\left[\sum_{j=1}^{n} z_{j} x_{j}(t)-\sum_{i=1}^{m} r_{i}(t)\right] \rightarrow \max , \\
r_{i}(t) \geq 0, \quad \sum_{i=1}^{m} r_{i}(t) \leq R, \quad t \in[0, T], \quad i=1,2, \ldots, m, \\
x_{*} \leq \sum_{j=1}^{n} z_{j} x_{j}(t) \leq x^{*}, \quad t \in[0, T], \\
J_{i}=\sum_{t=0}^{T} \delta^{t}\left[\sum_{j=1}^{n}\left(z_{j} x_{j}(t)-s_{j}^{i} u_{j}^{i}(t)\right] \rightarrow \max ,\right. \\
s_{j}^{i}= \begin{cases}1, & \text { if } \quad b_{j}^{i}>0, \\
0, & \text { if } \quad b_{j}^{i}=0,\end{cases} \\
\sum_{j=1}^{n} u_{j}^{i}(t) \leq r_{i}(t), \quad u_{j}^{i}(t) \geq 0, \quad t \in[0, T], \quad i=1,2, \ldots, m, \\
\dot{x}_{j}(t)=\sum_{i=1}^{m} b_{j}^{i} \sqrt{u_{j}^{i}(t)}+\sum_{l=1}^{n} a_{l j} x_{l}(t), \quad x_{j}(0)=x_{j 0}, \quad j=1,2, \ldots, n .
\end{gathered}
$$

Here $a_{i j}$ is a coefficient of influence of the $i$-th basic agent to the $j$-th basic agent in the discrete model. As earlier, when we move from the discrete description to the continuous one, the influence matrix $A$ is substituted by the matrix $\tilde{A}$ in the form

$$
\tilde{A}=A-I \text {. }
$$

Therefore,

$$
\tilde{a}_{i j}= \begin{cases}a_{i j}, & \text { if } \quad i \neq j \\ a_{i j}-1, & \text { if } \quad i=j .\end{cases}
$$

Let

$$
Z=\left(\begin{array}{c}
z_{1} \\
z_{2} \\
\ldots \\
z_{n}
\end{array}\right), z_{j}>0, j=1,2, \ldots, n
$$


be the Frobenius eigenvector of the matrix $A$, corresponding to the eigenvalue 1 of the matrix $A$. The diagonal matrix

$$
\Xi=\left(\begin{array}{cccc}
z_{1} & 0 & \ldots & 0 \\
0 & z_{2} & \ldots & 0 \\
\ldots & \ldots & \ldots & \ldots \\
0 & 0 & \ldots & z_{n}
\end{array}\right)
$$

is the matrix of transfer to the stochastic (from the right) matrix $P$ :

$$
P=\Xi^{-1} A \Xi .
$$

Then

$$
A=\Xi P \Xi^{-1},
$$

i.e.

$$
A^{\tau}=\Xi^{-1} P^{\tau} \Xi
$$

We can reformulate the problem (86)-(89) other coordinates. Introduce a family of matrices $B^{i}$ :

$$
B^{i}=\left(\begin{array}{cccc}
b_{1}^{i} & 0 & \ldots & 0 \\
0 & b_{2}^{i} & \ldots & 0 \\
\ldots & \ldots & \ldots & \ldots \\
0 & 0 & \ldots & b_{n}^{i}
\end{array}\right)
$$

and a family of vector functions

$$
\sqrt{U^{i}(t)}=\left(\begin{array}{c}
\sqrt{u_{1}^{i}(t)} \\
\sqrt{u_{2}^{i}(t)} \\
\ldots \\
\sqrt{u_{n}^{i}(t)}
\end{array}\right), i=1,2, \ldots, m .
$$

Then the constraint (89) takes the matrix form

$$
\dot{X}(t)=\sum_{i=1}^{m} B^{i} \sqrt{U^{i}(t)}+\left(\Xi^{-1} P^{\tau} \Xi-I\right) X(t),
$$

i.e.

$$
\dot{Y}(t)=\sum_{i=1}^{m} \tilde{B}^{i} \sqrt{U^{i}(t)}+\left(P^{\tau}-I\right) Y(t),
$$

where

$$
\begin{aligned}
& X(t)=\left(\begin{array}{c}
x_{1}(t) \\
x_{2}(t) \\
\ldots \\
x_{n}(t)
\end{array}\right), \quad Y(t)=\left(\begin{array}{c}
y_{1}(t) \\
y_{2}(t) \\
\ldots \\
y_{n}(t)
\end{array}\right)=\Xi X(t), \\
& \dot{X}(t)=\left(\begin{array}{c}
\dot{x}_{1}(t) \\
\dot{x}_{2}(t) \\
\ldots \\
\dot{x}_{n}(t)
\end{array}\right), \quad \dot{Y}(t)=\left(\begin{array}{c}
\dot{y}_{1}(t) \\
\dot{y}_{2}(t) \\
\ldots \\
\dot{y}_{n}(t)
\end{array}\right)=\Xi \dot{X}(t),
\end{aligned}
$$




$$
\tilde{B}^{i}=\Xi B^{i}=\left(\begin{array}{cccc}
z_{1} b_{1}^{i} & 0 & \ldots & 0 \\
0 & z_{2} b_{2}^{i} & \ldots & 0 \\
\ldots & \ldots & \ldots & \ldots \\
0 & 0 & \ldots & z_{n} b_{n}^{i}
\end{array}\right)=\left(\begin{array}{cccc}
\tilde{b}_{1}^{i} & 0 & \ldots & 0 \\
0 & \tilde{b}_{2}^{i} & \ldots & 0 \\
\ldots & \ldots & \ldots & \ldots \\
0 & 0 & \ldots & \tilde{b}_{n}^{i}
\end{array}\right), \quad \tilde{P}=P-I,
$$

or in coordinates:

$$
\dot{y}_{j}(t)=\sum_{i=1}^{m} \tilde{b}_{j}^{i} \sqrt{u_{j}^{i}(t)}+\sum_{l=1}^{n} p_{l j} y_{l}(t) .
$$

The constraint (87) takes the form

$$
x_{*} \leq \sum_{j=1}^{n} y_{j}(t) \leq x^{*}, \quad t \in[0, T] .
$$

The expression (86) is written as

$$
J_{0}=\int_{t=0}^{T} e^{-\rho t}\left[\sum_{j=1}^{n} y_{j}(t)-\sum_{i=1}^{m} r_{i}(t)\right] d t \rightarrow \max ,
$$

and the expression (88) as

$$
J_{i}=\int_{t=0}^{T} e^{-\rho t}\left[\sum_{j=1}^{n}\left(y_{j}(t)-s_{j}^{i} u_{j}^{i}(t)\right] d t \rightarrow \max .\right.
$$

Other expressions in the formulation of the problem (86)-(89) do not change.

Based on the previous solution, we find the optimal strategies of all firms:

$$
u_{j}^{i}(t)=\frac{\left(\tilde{b}_{j}^{i}\right)^{2} r_{i}(t)}{\sum_{j=1}^{n}\left(\tilde{b}_{j}^{i}\right)^{2}}=\frac{\left(z_{j} b_{j}^{i}\right)^{2} r_{i}(t)}{\sum_{j=1}^{n}\left(z_{j} b_{j}^{i}\right)^{2}}, \quad i=1,2, \ldots, m, \quad t \in[0, T],
$$

and the principal:

$$
\begin{gathered}
r_{i}(t)=\frac{\sum_{j=1}^{n}\left(z_{j} b_{j}^{i}\right)^{2}}{\sum_{k=1}^{m} \sum_{j=1}^{n}\left(z_{j} b_{j}^{k}\right)^{2}} \min \left\{\frac{1}{4}\left(\alpha^{0}(t)\right)^{2} \sum_{k=1}^{m} \sum_{j=1}^{n}\left(z_{j} b_{j}^{k}\right)^{2}, R\right\}, \quad t \in[0, h], \\
r_{i}(t), \quad t \in(h, T], \quad i=1,2, \ldots, m,
\end{gathered}
$$

where

$$
\alpha^{0}(t)=\frac{1}{\rho}\left(1-e^{\rho(t-h)}\right), \quad t \in[0, h] .
$$

The principal's maximal guaranteed result is equal to

$$
\begin{gathered}
\frac{1}{\rho}\left(e^{-\rho h}-e^{-\rho T}\right) x^{*}+\frac{1}{\rho}\left(1-e^{-\rho h}\right) x_{0}+ \\
+\int_{0}^{h}\left[\alpha^{0}(\tau)\left(\sum_{i=1}^{m} \sqrt{r_{i}(\tau) \sum_{j=1}^{n}\left(z_{j} b_{j}^{i}\right)^{2}}\right)-\sum_{i=1}^{m} r_{i}(\tau)\right] e^{-\rho \tau} d \tau,
\end{gathered}
$$


where the instant of time $h$ solves the equation

$$
y(t)=x^{*}=\int_{0}^{t} \sum_{i=1}^{m} \sqrt{r_{i}(\tau) \sum_{j=1}^{n}\left(z_{j} b_{j}^{i}\right)^{2}} d \tau+\sum_{j=1}^{n} z_{j} x_{j 0},
$$

i.e. $h$ is the instant $t$ when the right hand side of (90 becomes equal to $x^{*}$.

As soon, $S C I=1$ as in the previous problem from the Section 4 . The expressions for the maximal guaranteed payoff in this case differ from those in the section 4 only by the multipliers $z_{j}$ - the components of Frobenius eigenvector of matrix $A$. So, the interests of players are completely compatible as in the problem without the conditions of homeostasis.

\section{Conclusion}

We studied difference and differential Stackelberg game theoretic models of opinion control in marketing networks in which the principal should provide that a given limit value of the summary opinion not be exceeded. First, the problem is solved for the stochastic influence matrix A, and then this strong assumption is substituted by a weaker standard assumption that the matrix $\mathrm{A}$ is stochastic from the left. It is possible to introduce a weaker assumption, namely that a non-negative influence matrix has a strictly positive Frobenius eigenvector. In this case, it is only required to introduce in the received formulas the Frobenius eigenvalue of the influence matrix as a multiplier. It appears that for the principal it is not advantageous to allocate to the forms more resources than they need in their rational behavior. In all cases in frame of the considered model the interests of players are completely compatible.

Other formulations of the homeostasis conditions are possible, for example, $x_{*} \leq$ $\sum_{j=1}^{n} x_{j} \leq x^{*}$. But in the considered model, when all state variables can only increase their values or remain constant, the constraints from below are satisfied automatically.

Another formulation can use the constraints only for the terminal values of the variables, i.e. $x_{*} \leq \sum_{j=1}^{n} x_{j}^{T} \leq x^{*}$, where $x_{j}^{T}$ are the values of the state variables in the end of the game. However, the satisfaction of these conditions in any instant along the game implies their satisfaction in the final instant also. The inverse implication is also true because the values of the state variables do not decrease during the game.

At last, the constraints can bound each state variable separately: $x_{* j} \leq x_{j} \leq x^{* j}$ or $x_{* j} \leq x_{j}^{T} \leq x^{* j}$. In such formulation it is necessary to evaluate in which instant of time which variable reaches its boundary value. Starting from these instants, the control impact must be exerted only to the remaining state variable which decreases the problem dimension.

\section{References}

Agieva, M. T., Korolev, A. V., Ougolnitsky, G. A. (2019). Modeling and Simulation of Impact and Control in Social Networks. In: Modeling and Simulation of Social-Behavioral Phenomena in Creative Societies. First International EURO Mini Conference, MSBC 2019 Vilnius, Lithuania, September 18-20, 2019 Proceedings. Springer, 2019. P. 29-40.

Chkhartishvili, A., Gubanov, D., Novikov, D. (2019). Social Networks: Models of Information Influence, Control, and Confrontation. Springer Publishers. 
Leontief, W. (1987). Input-Output Analysis. The New Palgrave. A Dictionary of Economics, edited by J. Eatwell, M. Milgate, and P. Newman, vol. 2, pp. 860-864. London. Macmillan.

Nisan, N., Roughgarden, T., Tardos, E., Vazirani, V. (2007). Algorithmic Game Theory. Camdridge University Press.

Ougolnitsky, G. (2011). Sustainable Management. N.Y.: Nova Science Publishers.

Sedakov, A., Zhen, M. (2019). Opinion Dynamics Game in Social Network with Two Influence Nodes. Vestnik SPb Gos. Univ. Ser. Appl. Math. Inform. Sci. Contr. Proc., 15(1), 118-125.

Sraffa, P. (1960). Production of Commodities by Means of Commodities. Prelude to a Critique of Economic Theory. Cambridge: Cambridge University Pres.

Sraffa, P. (1962). Production of Commodities by Means of Commodities: A Comment. Economic Journal, 72, 477-479.

Sukhinov, A. I., Ougolnitsky, G. A., Usov, A. B. (2020). Methods of Solving the Theoretic Game Models for Coordinating Interests in Regulating the Fishery Industry. Mathematical Models and Computer Simulations, 12(2), 176-184.

Zhen, M. (2019). Stackelberg Equilibrium in Opinion Dynamics Game in Social Network with Two Influence Nodes. Contributions to Game Theory and Management. Vol. XII. Collected Papers of the Twelfth International Conference "Game Theory and Management", Petrosyan, L., and Zenkevich, N., Eds., St. Petersburg: Saint Petersburg State University, 366-386. 